Aus der Ohrenabteilung (Vorstand Prof. Dr. G. Alexander) und der Abteilung für Kinderheilkunde (Dozent Dr. L. Jehle) der Allgemeinen Poliklinik in Wien.

\title{
Über den Einfluß vasomotorischer Störungen im Kindesalter auf das Gehörorgan.
}

\author{
Von \\ Dr. Conrad Stein, Assistenten der Ohrenabteilung, \\ und
}

Dr. Rudolf Pollak, Assistenten der Abteilung für Kinderkrankheiten.

Es ist das Verdienst Hamburgers, das Symptomenbild der vasomotorischen Störungen im Kindesalter unter $\mathrm{Zu}$ grundelegung klinischer Beobachtungsergebnisse in prägnanter Weise dargestellt und uns durch genaue Schilderung der Wirkungen vasomotorischer Übererregbarkeit auf den kindlichen Organismus die Wichtigkeit ihrer Kenntnis veranschaulicht zu haben.

Hamburger hat darauf hingewiesen, daß die erhöhte Erregbarkeit des Herzens und des Gefäßapparates in gleicher Weise wie bei nervösen und neurasthenischen Erwachsenen auch im Kindesalter auftreten kann, und daß aus der labilen Innervation des Zirkulationsapparates eine Reihe bedeutsamer Krankheitssymptome resultiert. Diese Symptome werden teils vom Herzen, teils vom Gefäßapparate ausgelöst und sind sowohl subjektive wie objektive.

Als subjektive Erscheinungen von seiten des Herzens nennt Hamburger: schon nach geringen körperlichen Anstrengungen und psychischen Erregungen auftretendes Herzklopfen und unangenehme Sensationen in der Herzgegend (Stechen, Druckgefühl, mitunter mehr weniger heftige Schmerzen, die auch mit dem Gefühle der Atemnot einhergehen können), als objektive Symptome: hebenden, etwas verbreiterten Spitzenstoß, leichte epigastrische Pulsation, gar keine oder nur geringe Herzdilatation, größere oder geringere Verschieblichkeit des Herzens bei Lagewechsel und auffallend 
labilen Rhythmus der Herzaktion (starke Beeinflußbarkeit der Schlagfolge durch leichte körperliche Anstrengungen, Pulsus irregularis respiratorius, etc.) bei reinen Herztönen.

Von subjektiven Gefäßs ym pto men erwähnt Hambur ger Kopfschmerzen, die besonders im Anschluß an längerdauernde geistige Anstrengungen auftreten und auf Zirkulationsstörungen (Hyperämie oder Ischämie) zurückzuführen sind und aus einer Ischämie resultierendes Schwindelgefühl. Objektiv sich bemerkbar machende Symptome von seiten des Gefäßapparates sind leichtes Erröten oder Erblassen bei psychischen Erregungen, kalte und feuchte $\mathrm{Hände}$ und Füße, Dermographie, sichtbares Klopfen der Karotiden, erhöhter Tonus der Arterienwand an den Radiales und Temporales. Hamburger hat ganz besonders auf das bei Kindern gänzlich unbeachtete Symptom der Rigidität der Arterien hingewiesen und gezeigt, daß diese (von Krehl, Romberg und Schlayer bei juvenilen Individuen wiederholt beobachtete Erscheinung) lediglich auf einen erhöhten Tonus der Gefäßmuskulatur zurückzuführen sei.

Die vasomotorischen Störungen im Kindesalter haben nach Hamburger ihre Ursache in einer allgemeinen Schwäche des ganzen Innervationsapparates für Herz und Gefäße (Angiasthenie oder Kyklasthenie), vermöge welcher schon geringfügige Reize pathologische Erscheinungen von seiten des Zirkulationsapparates auslösen können.

Unter den Faktoren, die auf der Grundlage dieser hypothetischen angeborenen Angiasthenie krankhafte Störungen hervorrufen, stehen im kindlichen Alter psychische Traumen, geistige Anstrengungen und seelische Erregungen an erster Stelle; auch der Einwirkung von Bakterientoxinen dürfte vielleicht ein Einfluß auf den Zirkulationsapparat zuzusprechen sein. Selbstverständlich kann jedes Moment, das das Zirkulationssystem durch Nervenschädigung zu beeinträchtigen vermag, krankhafte Innervationsstörungen bedingen.

Das Krankheitsbild, das sich aus der vasomotorischen Übererregbarkeit ergibt, ist je nach der Lokalisation der befallenen Gefäßbezirke ein verschiedenes.

Am häufigsten begegnen wir Klagen der erkrankten Kinder über Kopfschmerzen, Schwindelgefühl und Ohnmachtsanwandlungen, Erscheinungen, die wahrscheinlich in einer Störung der Zirkulation in den zerebralen Gefäßen begründet sind. In vielen Fällen stehen die Herzerscheinungen im Vordergrunde des Krankheitsbildes. Eine Krankheitserscheinung, die $\mathrm{Ham}$ burger bei vasomotorischer Übererregbarkeit zu konstatieren 
Gelegenheit hatte, ist die orthotische, beziehungsweise lordotische Albuminurie, und ohne die Richtigkeit der mechanischen Theorie Jehles anzuzweifeln, meint $\mathrm{Hamburger,} \mathrm{daß} \mathrm{auch}$ vasomotorischen Einflüssen eine ätiologische Bedeutung für die Entwicklung dieses Symptoms zuerkannt werden müsse.

Ebenso dürfte das Asthma bronchiale bei Kindern nach Hamburger nicht selten auf vasomotorische Störungen zurückzuführen und aus einer Hyperämie der Lungenkapillaren zu erklären sein.

Wir sehen also, daß verschiedene Organe dadurch, daß der Vasomotorenapparat schon auf geringfügige Reize mit Störungen reagiert und eine abnorme Blutverteilung, sei es im Sinne einer Vasodilatation, sei es einer Vasokonstriktion, veranlaßt, funktionell in mehr oder weniger ausgesprochener Weise beeinträchtigt werden können.

Es mußte nun angesichts der durch vielfache Erfahrungen erhärteten Tatsache, daß das Gehörorgan sich Zirkulationsstörungen gegenüber als ganz besonders empfindlich erweist, von Interesse sein, Kinder mit vasomotorischen Störungen auch auf das Bestehen von Krankheitserscheinungen seitens des Hörapparates zu untersuchen. Der Gedanke an die Durchführung von Untersuchungen in dieser Richtung erschien um. so naheliegender, als der Einfluß vasomotorischer Zirkulationsstörungen auf das Gehörorgan durch die Ergebnisse einer Reihe otiatrischer Beobachtungen an Erwachsenen vollkommen außer Zweifel gestellt worden ist.

So erwähnt Politzer unter den funktionellen Lähmungszuständen des Hörnerven, die mit Herabsetzung oder Aufhebung der Hörfunktion verbunden sind, auch eine a ngio neurotische Akustikuslähmung, die durch plötzliches Erblassen des Gesichtes, UUbelkeit, Schwindel, Ohrensausen und Schwerhörigkeit charakterisiert ist. Diese Symptome verschwinden nach einigen Minuten mit dem Eintritte der früheren. normalen Gesichtsfarbe vollständig, ohne auch nur den geringsten Grad von Hörstörung zu hinterlassen (Brunner).

Nach V. Urbantschitsch dürften auch die bei Migräne vorkommenden Hörstörungen zum großen Teile auf vasomotorische Störungen im Gebiete der akustischen Zentren zurückzuführen sein.

Auch die Motionstaubheit dürfte in gleichem Sinne zu deuten séin. In einem von Urbantschitsch beobachteten Falle waren linksseitig plötzlich hochgradige Schwerhörigkeit, Ohrensausen, Herabsetzung der Geruchs-, Geschmacks- und Tast- 
empfindung als bleibende Symptome, ferner vorübergehend Skotome und Sehschwäche infolge von Schreck aufgetreten. Amylnitrit rief auf der rechten Seite eine lebhafte Röte hervor, indes sich die linke Gesichtshälfte nur schwach gerötet zeigte; nach Urbantschitsch waren vielleicht die Sinnesstörungen in diesem Falle durch einen Gefäßkrampf im Gebiete der betreffenden Sinneszentren bedingt.

Urbantschitsch zitiert die Beobachtungen von $B$ ur nett, der drei Fälle von Ohrensausen und Schwerhörigkeit mit bedeutender Röte der das $\mathrm{Ohr}$ umgebenden Partien beschreibt, die Mitteilung Wendts, der in einem Falle nach einem Fußbade das Auftreten von Taubheit und Ohrgeräuschen beobachtete und die Angaben Itards, der von einem Falle berichtet, in welchem regelmäßig während der Verdauungsperiode hochgradige Schwerhörigkeit und heftige Ohrgeräusche eintraten.

Auch das rasche Schwinden und Wiedererscheinen mancher Pulsationsgeräusche im Ohre dürfte nach Urbantschitsch durch vasomotorische Einflüsse bedingt sein.

In jüngster Zeit hat $\mathrm{O}$. Mayer unter Zugrundelegung klinischer Beobachtungen und anatomischer Befunde die Ansicht vertreten, daß die anatomischen Veränderungen der Otosklerose durch Zirkulationsstörungen veranlaßt werden. Als Ursache für die Entwicklung des Krankheitsprozesses im Ohre scheinen ihm vor allem Störungen der Funktion des vasomotorischen Nervensystems in Betracht zu kommen. Durch lokale Zirkulationsstörungen im Gebiete der Endausbreitung der Arteriae nutritiae (Verengerung der zuführenden Gefäße und mangelhafte Blutzufuhr) werden nach Mayer herdförmige Erkrankungen des Knochens der Pyramide hervorgerufen. Mayer hält es für wahrscheinlich, daß auch andere Ursachen lokaler Zirkulationsstörungen, so insbesondere organische Gefäßerkrankungen (Arteriosklerose) dieselben Veränderungen hervorrufen können.

Mayer verweist auch auf eine Reihe klinischer Beobachtungen, aus welchen hervorgeht, daB die Symptome mancher Otosklerotiker im Ohre auf eine Störung der Funktion des Vasomotorenapparates bezogen werden müssen. So hat Bing auf die bei Otosklerotikern fehlende Ceruminalabsonderung, Hammerschlag auf eine verminderte Schweißsekretion hingewiesen. Urbantschitsch führt auch die von Fröschels beobachtete Herabsetzung des Kitzelgefühles im Gehörgange auf vasomotorisch-trophische Einflüsse zurück. Auch Sohier Bryant betrachtet die Zirkulationsstörun- 
gen als einen der wichtigsten sekundären Faktoren in der Ätiologie der Otosklerose. Als Grundlage der Zirkulationsstörung liegt eine wirksame Ursache vor in der Störung der vasomotorischen Innervation und der inneren Sekretion im weitesten Sinne. Auch die Arteriosklerose ist nach Sohier Bryant eine mögliche Ursache der Otosklerose infolge der durch sie bedingten Ernährungsschädigung. Wie bei Anämie und Chloroseliefert die Arteriosklerose eine mangelhafte Blutversorgung; die Knochenveränderungen werden daher als trophoneurotische bezeichnet.

Delmarcel und Dels ta nche berichteten auf demKongresse der belgischen Gesellschaft für Otologie und Rhinologie in Brüssel (13. bis 14. Juli 1912) über die Ursache von Ohrensausen und die Pathogenese der Ohrgeräusche und betonten hauptsächlich auch die Rolle der vasomotorischen Störungen und die gefäßverengernde Wirkung des Sympathikus.

Die Möglichkeit der Einwirkung vasomotorischer Zirkulationsstörungen finden wir auch bei Wittmaac $\mathrm{k}$ angeführt, der in seiner Arbeit über die toxische Neuritis acustica die Ansicht ausspricht, daß auch derartige leichte Zirkulationsstörungen imstande sind, die schädigenden Einflüsse von Giftwirkungen auf den Akustikus zu unterstützen.

C. Stein, der sich seit vielen Jahren in eingehender Weise mit dem Studium des Einflusses von Zirkulationsstörungen auf das Gehörorgan beschäftigt, bespricht in einer vor kurzem erschienenen Arbeit an der Hand eines reichen Beobachtungsmaterials die große Bedeutung pathologischer Zirkulationsverhältnisse für die Entstehung subjektiver Ohrgeräusche und weist bei dieser Gelegenheit auf die Wirkungen vasomotorischer Störungen auf das Gehörorgan hin.

Nach seinen Erfahrungen können sich die Folgeerscheinungen der vasomotorischen Neurosen so wie in anderen Gebieten auch in jenem des Gehörorganes in vorübergehenden, aber auch in bleibenden Störungen der Funktion bemerkbar machen.

Die vasomotorischen Störungen können alle Anteile des Gehörorganes (äußeres, mittleres und inneres Ohr) betreffen; die durch sie veranlaßten pathologischen Erscheinungen werden in verschiedener Weise zustandekommen, je nachdem es sich um Erregungs- oder Lähmungszustände der Vasokonstriktoren, resp. - dilatatoren handelt. Die Störungen im Gebiete der Gefäßnerven können nach Ansicht Steins zunächst zur Entstehung von Ohrgeräuschen Veranlassung geben. Dies kann auf zweifache Weise geschehen: 1. dadurch, daß vasokon- 
striktorische, resp. vasodilatatorische Zustände auf den Akustikus einwirken (die Vasokonstriktion durch eine verringerte Blutzufuhr zum Hörnervengebiete, die Vasodilatation durch Vermittlung von GefäBgeräuschen, die auf dem Wege verstärkter Kopfknochenleitung zur Perzeption gelangen), und 2. dadurch, daß Erregungszustände des vasomotorischen Nervensystems (durch Vasokonstriktion) zu einer erhöhten Tätigkeit des Herzens mit Steigerung des Blutdruckes führen, die ihrerseits durch verstärkte Pulsation des Blutstromes in den Gefäßen des Ohres oder seiner Umgebung oder durch verstärkte Pulsation des Liquor cerebrospinalis (vgl. Stein, Utber die Zirkulationsverhältnisse im Schädelinneren bei venöser Stauung und ihre Beziehungen zu den Ohrgeräuschen, Arch. f. Ohrenh. 1911, Bd. 86) Gehörsempfindungen verursacht.

Von besonders nachteiliger Wirkung auf den Hörapparat ist die Vasokonstriktion; sie führt zunächst zu einem Erregungszustande des Hörnerven, wenn jedoch die Blutzufuhr nicht wieder in normaler Weise erfolgt, zu einem degenerativen Prozesse des kochlearen Neurons. Im Gegensatze zu der Arteriosklerose, welche dauernd eine Verengerung bestimmter Gefäßbezirke hervorruft, kann die bei funktionellen Erkrankungen des Gefäßsystems temporär eintretende, aus Vasomotorenwirkungen resultierende abnor me Blutverteilung immer wieder regulatorisch ausgeglichen werden. Immerhin muB daran festgehalten werden, daß sich der Akustikus Zirkulationsstörungen gegenüber als überaus empfindlich erweist und daß eine häufig wechselnde Blutverteilung in seinem Stromgebiete, wie sie durch ein reges Spiel der Vasomotoren verursacht wird, zunächst zu Erregungszuständen, weiterhin aber zu nutritiven Störungen im Akustikus führt.

Im nachfolgenden sollen die Resultate otiatrischer Untersuchungen an einer Reihe von Kindern, bei denen die subjektiven und objektiven Krankheitssymptome einer erhöhten vasomotorischen Erregbarkeit erhoben werden konnten, mitgeteilt werden.

Die Zahl der von uns untersuchten Kinder (die anamnestischen Details und klinischen Befunde sind am Schlusse der Arbeit bekanntgegeben) beträgt 40 .

Von den untersuchten Kindern (14 Mädchen und 26 Knaben) standen 1 im Alter von 5 Jahren,

$\begin{array}{llllll}1 & , & , & , & 6 & , \\ 4 & , & , & & 7 & \\ 5 & , & , & , & 8 & ,\end{array}$




\begin{tabular}{|c|c|c|c|}
\hline im & Alte & von 9 & Jahr \\
\hline 4 , & " &, 10 & ", \\
\hline , & $\because$ &, 11 & , \\
\hline 7, & , &, 12 & \\
\hline " &, & , 13 & \\
\hline
\end{tabular}

Wir haben ausschließlich solche Fälle zu unseren Untersuchungen herangezogen, bei denen die Innervationslabilität des $\mathrm{Ge}$ fäßsystems durch die von $\mathrm{Hamb}$ urger angeführten charakteristischen Krankheitserscheinungen in klarer und typischer Weise gekennzeichnet war.

Die Mehrzahl der Kinder wurde wegen verschiedener Krankheitssymptome von seiten des Zirkulationsapparates, der Atmungsorgane und des Verdauungsa pparates zur internen Untersuchung gebracht, und erst der Nachweis der vasomotorischen Störungen bot die Veranlassung zur Untersuchung des Ohres. Die Minderzahl kam wegen Beschwerden seitens des Ohres zunächst zur ohrenärztlichen Untersuchung und wurde erst nachher wegen anamnestisch erhobener Krankheitserscheinungen in anderen Organen intern untersucht.

Vier Fälle (Nr. 4, 12, 16, 30), in denen keine subjektiven Beschwerden angegeben wurden und ein in jeder Richtung (otoskopisch und funktionell) absolut normaler Befund erhoben wurde, konnten als vollständig normal bezeichnet werden.

In einem Falle (Nr. 19) wurde eine einseitige chronische Mittelohreiterung (bei normalem Befunde auf dem anderen Ohre) festgestellt. Das erkrankte Ohr ergab ausschließlich Krankheitserscheinungen, welche der vorliegenden Mittelohrerkrankung entsprachen und wies weder im subjektiven noch im objektiven Krankheitsbilde irgendwelche Besonderheiten auf.

In drei Fällen (Nr. 11, 17, 36) fanden sich beiderseits katarrhalische Veränderungen im Mittelohre als Folgeerscheinungen adenoider Vegetationen. Die Hörstörungen, die in diesen Fällen nachweisbar waren, konnten durch Luftduschen vorübergehend und durch die Adenotomie dauernd zum Schwinden gebracht werden. Ebenso schwand nach der Beseitigung des ursächlichen Momentes des Mittelohrkatarrhs das Ohrensausen, über das eines dieser drei Kinder klagte*).

*) Zwei der hier erwähnten Fälle zeigten das im nachfolgenden besprochene Ermüdungsphänomen; dieses blieb auch nach dem Rückgange der katairhalischen Erscheinungen im Mittelohre unverändert fortbestehen. 
Neun Kinder (Nr. 5, 7, 13, 14, 15, 21, 22, 37, 38) mit normalem oder nur unwesentlich von der Norm abweichendem otoskopischen Befunde (leichte Retraktion, geringe partielle Trübung des Trommelfelles) und vollständig normaler Hörweite boten in eklatanter Weise das von Hammersohlag als Ermüdungsphänomen beschriebene Symptom dar.

Hammerschlag wies darauf hin, daß der funktionell geschädigte, aber nur in geringem Grade hypästhetische Akustikus für kontinuierliche, dabei in ihrer Intensität allmählich abschwellende Schallreize, wie es Stimmgabeltöne sind, abnorm leicht ermüdet. Diese Prüfung auf das „Ermüdungsphänomen" wird in der Weise vorgenommen, daß eine Stimmgabel von mittlerer Höhe ad maximum angeschlagen und so lange vor das zu prüfende Ohr gehalten wird, bis der Patient angibt, den Ton nicht mehr zu hören. Die Stimmgabel wird nun vom Ohre entfernt und nach 2-3 Sekunden, ohne neuerlich angeschlagen worden zu sein, dem Ohre wieder genähert. Der Patient gibt jetzt an, den Ton wieder zu hören, und er hört ihn in der Regel wieder einige Sekunden lang. Mitunter läßt sich der Versuch noch ein zweites- und ein drittesmal wiederholen.

Dieser Stimmgabelbefund konnte bei 9 Kindern mit im übrigen vollkommen normalem Gehörorgane und ausgezeichnetem Hörvermögen und bei zweien der Kinder, die katarrhalische Veränderungen im Mittelohre zeigten, in mehr oder weniger deutlich ausgeprägter Weise festgestellt werden. Das Ermüdungsphänomen war in allen Fällen beiderseits und zwar bei den meisten Kindern dreimal nacheinander zu beobachten. Die nicht mebr gehörte Stimmgabel wurde nach einer Pause von einigen Sekunden nochmals durch $6-8$ Sekunden und nach einer zweiten, resp. dritten gleichlangen Pause wiederum mehrere Sekunden hindurch perzipiert. Die Forderung Hammerschlags, man dürfe diesem Symptom nur dann eine pathognostische Bedeutung zuschreiben, wenn die erste Ermüdungspause noch in die Zeit fällt, da die Stimmgabel noch sehr deutlich tönt, war in allen beobachteten Fällen erfüllt.

Bei zwei Kindern, bei denen innerhalb eines größeren Zeitraumes wiederholte Untersuchungen vorgenommen werden konnten, zeigte sich - dem Allgemeinbefinden der Patienten entsprechend - ein nicht unwesentlicher Wechsel im Befunde: An Tagen, an denen sich die Kinder besser befanden, war das Ermüdungsphänomen in geringem Grade, an Tagen, an denen die Kinder matt und müde waren, nach schlecht verbrachter

Archiv f. Ohrenheilkunde, Bd. 96. 
Nacht, bei gleichzeitig bestehenden Kopfschmerzen usw. in stärkerem Grade, d. h. in häufigerer Wiederholung, festzustellen. Das Ermüdungsphänomen zeigte sich in allen Fällen, in denen es auf dem Wege der Luftleitung nachweisbar war, auch auf dem Wege der Kopfknochenleitung; hier kam es allerdings, der kürzeren Dauer derselben entsprechend, weniger oft zur Feststellung.

Bei 19 Kindern (den Fällen Nr. 1, 2, 3, 6, 8, 9, 10, 18, 24, $25,27,28,29,32,33,34,35,39$ und 40) ergab die Funktionsprüfung des Gehörorganes die Symptome einer Erkrankung des Schallperzeptionsapparates, d. h. Verringerung der Hörschärfe und Verkürzung der Kopfknochenleitung bei positivem Rinne und Abnahme der Hördauer für hohe Töne.

In drei Fällen (Nr. 6, 18 und 24) war die Erkrankung des inneren Ohres eine einseitige und leichtgradige*),

in sechs Fällen (Nr. 9, 10, 25, 27, 34 und 35) war die Erkrankung eine beiderseitige und leichtgradige,

in vier Fällen (Nr. 1, 2, 32, und 33) war das eine $\mathrm{Ohr}$ in geringem, das andere in mittlerem Grade*) affiziert,

in einem Falle (Nr. 3) fand sich auf der einen Seite eine mittel-, auf der anderen eine hochgradige*) Erkrankung des inneren Ohres,

in zwei Fällen (Nr. 8 und 29) konnte eine beiderseitige hochgradige Erkrankung des inneren Ohres,

in drei Fällen (Nr. 28, 39 und 40) einseitige Taubheit (nebst geringgradiger Erkrankung des Schallperzeptionsapparates der anderen Seite in zwei Fällen und mittelgradiger in einem Falle) konstatiert werden.

Bemerkenswert ist, daß bei drei Kindern, bei denen es möglich war, die Ergebnisse der Untersuchung durch wiederholte Prüfungen zu kontrollieren, Schwankungen der Hörschärfe festgestellt werden konnten in der Weise, daß das Hörvermögen für Flüstersprache und Taschenuhr an verschiedenen Tage differierte. Die Schwankungen der Hörschärfe gingen in unverkennbarer Weise parallel dem Allgemeinbefinden derart, daß das Hörvermögen besser war, wenn sich die

*) Die Bezeichnung ,leicht- oder geringgradige Erkrankung des inneren Ohres" bezieht sich auf Fälle, deren Hörvermögen nur in geringem Grade herabgesetzt war (Hörweite für eine normal $75 \mathrm{~cm}$ weit gehörte Taschenuhr $30-70 \mathrm{~cm}$, für Flüstersprache $8-10 \mathrm{~m}$ ), die Bezeichnung, „mittelgradige Frkrankung" auf Falle, in denen die Taschenuhr 5-30 cm weit, Flüstersprache 5-8 $\mathrm{m}$ weit gehört wurde, die Bezeichnung, hochgradige Frkrankung" anf walle mit noch geringerer Hörweite. 
Kinder wohler fühlten, und schlechter, wenn der Allgemeinzustand der Patienten ein ungünstigerer war. Die Differenzen der Hörweite betrugen für Flüstersprache - es wurden jedesmal dieselben Worte vorgesprochen $-2-4 \mathrm{~m}$, für die Taschenuhr bis zu $10 \mathrm{~cm}$.

In der gleichen Weise schwankten auch die mittelst der Stimmgabel erhobenen Befunde, indem die Hördauer für Stimmgabeltöne (Luft- und Knochenleitung), konform der Hörweite für Flüstersprache und Uhr, kleine Unterschiede aufwies.

Charakteristisch war auch der Einfluß länger fortgesetzter Hörprüfungen. Die Kinder ermüdeten bei längerer Dauer der Untersuchung ausnahmslos (manche ließen oft schon sehr frühzeitig Zeichen der Abspannung erkennen) und zeigten bei längerer Fortsetzung der Prüfung eine mitunter nicht unwesentlich geringere Hörweite, beziehungsweise Hördauer als im Beginne der Untersuchung. Es wurden unter Berücksichtigung dieser Tatsache immer nur die Anfangswerte der Hörprüfungen zum Vergleiche herangezogen.

Es muß jedoch betont werden, daß die objektiven Zeichen der Erkrankung des inneren Ohres (verkürzte Kopfknochenleitung, Verringerung der Hördauer für hohe Töne) - von den erwähnten graduellen Unterschieden abgesehen - bei allen Untersuchungen immer deutlich ausgesprochen waren, so daß an der Diagnose einer Erkrankung des Schallperzeptionsapparates festgehalten werden mutBe.

Eine Ausnahmsstellung darf jener Fall beanspruchen, den wir unter Nr. 23 unseres Beobachtungsmateriales anführen. Es handelte sich um einen 9 jährigen Knaben, bei welchem nach einer schweren Influenza stark ausgeprägte vasomotorische Erscheinungen (Kopfschmerzen, Schwindel, körperliche und geistige Ermüdbarkeit, Appetitlosigkeit usw.) zutage traten. Durch eine sich in auffälliger Weise bei dem Knaben geltend. machende Verringerung der Hörschärfe wurden die Eltern veranlaßt, ihn ohrenärztlich untersuchen zu lassen. Die Untersuchung ergab otoskopisch durchaus normalen Befund; bei der Funktionsprüfung fand sich eine beiderseitige Herabsetzung der Hörschärfe für Flüstersprache $(5 \mathrm{~m})$ und Taschenuhr $(25 \mathrm{~cm})$ bei leichter Verkürzung der Hördauer für $\mathrm{e}^{4}$ und $\mathrm{C}$ und Verringerung der Kopfknochenleitung (Vestibularapparat normal). Es bestanden also die charakteristischen Anzeichen einer Erkrankung des kochlearen Anteils des inneren Ohres. Lufteinblasungen änderten den Befund in keiner Weise. Es 
zeigten sich jedoch dem Allgemeinbefinden des Knaben entsprechend außerordentlich große Unterschiede im Funktionsbefunde. An Tagen, an denen das Allgemeinbefinden des Kindes ein schlechtes war (an solchen Tagen bestand außerordentliche Blässe des Gesichtes, das Kind zeigte große Mattigkeit und Hinfälligkeit und klagte über Kopfschmerzen), ergaben sich die vorhin genannten Zeichen der Erkrankung des inneren Ohres. An Tagen, an denen das Befinden des Knaben ein gutes war, war das Gehör vollständig normal, der Funktionsbefund bis auf ein ein- bis zweimal zu konstatierendes Ermüdungsphänomen ein normaler. Nach längerem Landaufenthalte, Einhaltung roborierender Diät und internem Gebrauche von Arsen kam das Kind bei bestem Wohlbefinden und ausgezeichnetem Aussehen wieder zur Untersuchung, die vollständig normale Verhältnisse feststellte. Wiederholte Nachprüfungen bestätigten das Ergebnis dieser Untersuchung.

Einen zweiten, dem hier beschriebenen an die Seite zu stellenden Fall teilen wir an anderer Stelle (S. 227 u. 228, Fall von $\mathrm{Dr}$. Leidler) in seinen Einzelheiten mit.

Elf unserer Patienten gaben an, Ohrgeräusche (Klingen Summen, Sausen usw.) zu hören. Es waren dies Fall 11 (akuter Mittelohrkatarrh), Fall 15 (normales $\mathrm{Ohr}$ ), Fall 20 (normales Ohr), Fall 21 (normales Ohr), Fall 23 (normales Ohr), Fall 25 (Erkr ankung des inneren Ohres), Fall 26 (normales Ohr), Fall 27 (Erkrankung des inneren Ohres), Fall 29 (Erkrankung des inneren Ohres), Fall 32 (Erkrankung des inneren Ohres) und Fall 35 (Erkrankung des inneren Ohres). An Ohrgeräuschen litten somit fünf Fälle mit funktionell normalem Hörapparate, fünf Fälle mit Erkrankung des inneren Ohres und ein Fall mit akut katarrhalischen Erscheinungen im Mittelohre. Während die Gehörsempfindungen in dem letztgenannten Falle nach Herstellung normaler Schalleitungsverhältnisse rasch und dauernd sistierten, blieben sie in einem Teile der übrigen Fälle lange Zeit, in einem Teile der Fälle dauernd bestehen. Es muß jedoch bemerkt werden, daB sie in keinem Falle unserer Beobachtung konstant bestanden, sondern nur zeitweise auftraten und ausnahmslos in Perioden schlechteren Allgemeinbefindens besonders heftig empfunden wurden. Als charakteristisch muß des weiteren betont werden, daß die Ohrgeräusche vor allem solche Fälle betrafen, bei denen Krankheitserscheinungen von seiten der zerebralen Gefäße bestanden. Auch fiel ihr Auftreten zeitlich mit dem Einsetzen von Kopfschmerzen und Schwindel überaus häufig zusammen. In einem Falle 
traten die subjektiven Gehörsempfindungen im Anschlusse an eine mehrwöchentliche eiterige Mittelohrentzündung, in einem anderen Falle unmittelbar nach einem Schlage auf das Ohr auf.

Zehn Kinder, und zwar fünf Fälle mit normalem Gehörorgan (Nr. 7, 13, 22, 31 und 38), vier Fälle mit Erkrankung des inneren Ohres (Nr. 2, 3, 18, 24) sowie ein Fall mit akuten katarrhalischen Veränderungen im Mittelohre klagten über schmerzhafte Sensationen im Gebiete des Ohres. Die schmerzhaften Empfindungen; welche die katarrhalischen Erscheinungen im Mittelohre im Falle Nr. 11 begleiteten, verschwanden vollständig, als nach der Entfernung der adenoiden Vegetationen die Symptome des Mittelohrkatarrhs zurückgingen. In den anderen Fällen handelte es sich um Schmerzen, die intermittierend - in zwei Fällen eínigemale im Tage, in den anderen Fällen im Verlaufe von Tagen oder Wochen nur ab und zu - auftraten und in der Dauer von einigen Minuten bis zu einer halben Stunde anhielten. Die Schmerzen, für welche sich im Ohre selbst und in seinen Nachbarorganen kein ursächliches Moment feststellen ließ, wurden in die Tiefe des Ohres, in die Gegend der Ohrmuschel, des Warzenfortsatzes, in die Schläfe-, Scheitel- oder Hinterhauptgegend verlegt. In einem Falle (Nr. 13) berichtete die Mutter des Kindes, dasselbe schreie ganz plötzlich auf und fahre sich mit den Händen an die Ohren oder hinter dieselben. Ein 10 jähriges Mädchen gab an, es fühle die Schmerzen so, ,als wäre etwas wund im Ohre".

Einen äußerst instruktiven Fall, in welchem die Art der Entstehung der Schmerzen durch die objektiven Begleiterscheinungen während des Anfalles in sehr eklatanter Weise gekennzeichnet wurde, verdanken wir der freundlichen Mitteilung unseres Kollegen Dr. Rudolf Leidler.

Die Krankengeschichte betrifft ein 7 jähriges Mädchen (Mutter und ein Bruder sehr nervös und anämisch) mit allgemeinen, deutlich ausgesprochenen vasomotorischen Krankheitserscheinungen. Die Mutter erzählt, das Kind leide öfters an Anfällen von heftigen Schmerzen im rechten Ohre, die vom Ohreingange ausgehend, einerseits gegen die Scheitel- und Hinterhauptgegend und andererseits den Hals entlang ausstrahlen. Die Schmerzen dauern mitunter einen ganzen Tag lang an und wiederholen sich zwei- bis dreimal in der Woche. Zur Zeit der Schmerzen, während welcher das Kind Ohrensausen empfindet und schlecht hört, ist es auffallend blaß and vollständig appetitlos. 
Die Temperatur ist zur Zeit der Schmerzanfälle vollständig normal. In der schmerzfreien Zeit befindet sich das Kind vollständig wohl, doch bleibt jedesmal eine noch längere Zeit hindurch festzustellende Empfindlichkeit des Warzenfortsatzes zurück. Das Kind leidet seit Jahren an Oxyuris.

Herr Dr. Leidler hatte Gelegenheit, das Kind während eines solchen Schmerzanfalles zu untersuchen und erhob hier bei folgenden Befund: Das Kind, dessen Gesicht ungewöhnlich blaß ist und ein fast grünliches Kolorit zeigt, klagt über sehr große Ohrenschmerzen. Die leiseste Berührung des Warzenfortsatzes verursacht die heftigsten Schmerzen, so daß das Kind laut aufschreit. Hautdecke des Processus mastoideus vollständig normal, Trommelfell beiderseits normal. Hörweite links: Flüstersprache $10 \mathrm{~m}$, rechts: Flüstersprache $11 / 2-2 \mathrm{~m}$, Konversationssprache ea. 3-4 m. Nach Luftdusche keine Veränderung der Hörweite. Das Ansetzen der Stimmgabel am Warzenfortsatz wird außerordentlich schmerzhaft empfunden, so daB die Kopfknochenleitung, die scheinbar verkïrzt ist, nicht genau geprüft werden kann. Es zeigt sich jedoch eine auffallende Verkürzung für $\mathrm{e}^{4}$ (Luftleitung). Rhinoskopischer Befund: kleine adenoide Vegetationen, sonst keine Besonderheiten. Eine mehrere Wochen später in schmerzfreier Zeit wiederholte Untersuchung des Kindes ergab vollständiges Wohlbefinden, ausgezeichnetes Gehör beiderseits und durchaus normalen Stimmgabelbefund.

Der Bericht Leidlers zeigt den außerordentlichen Unterschied im Funktionsbefunde während einer Schmerzattacke und außerhalb einer solchen. Er belehrt uns darüber, daß gleichzeitig mit den Schmerzen die objektiven Symptome der Funktionsbeeinträchtigung im inneren Ohre festzustellen waren, während sich in der schmerzfreien Zeit das innere Ohr als funktionell vollkommen normal erwies.

Der Gleichgewichtsapparat, dessen Erregbarkeit in sämtlichen Fällen auf dem Drehstuhle und kalorisch geprüft wurde, zeigte in 36 Fällen ein durchaus normales Verhalten. Abweichungen von der Norm fanden sich in wesentlichem Grade in jenen drei Fällen, in denen die Untersuchung einseitige Taubheit ergeben hatte (Fall Nr. 28, 39 und 40).

Im Falle 28 (13jähriges Mädchen mit linksseitiger Taubheit) zeigte sich auf dem Drehstuhle ein deutlicher Unterschied zu ungunsten der linken Seite, bei der kalorischen Prüfung Unerregbarkeit des linken Vestibularapparates. Im Falle 39 (12jähriges Mädchen mit linksseitiger Taubheit) 
konnte gleichfalls eine Ausschaltung des Vestibularapparates des ertaubten Ohres festgestellt werden, und im Falle 40 (8jähriges Mädchen mit linksseitiger Taubheit) erwies sich der linke Vestibularapparat kalorisch in eklatanter Weise weniger erregbar als der rechte, während auf dem. Drehstuhle kein wesentlicher Unterschied zu konstatieren war.

Besonderes Interesse bot hinsichtlich des Verhaltens des statischen Apparates der Fall 24.

Er betraf ein 10jähriges Mädchen, das die Ohrenabteilung der Poliklinik mit der Klage über zeitweise auftretende heftige Schwindelanfälle mitÜbelkeit undBrechreiz und über häufig sich wiederholendes Ohrenstechen aufsuchte. An drei aufeinanderfolgenden Tagen wiederholte Untersuchungen ergaben stets den gleichen Befund: Rechtes Ohr normal, links : leichtgradige Erkrankung des inneren Ohres. Die interne Untersuchung ergab alle Zeichen einer bedeutenden vasomotorischen Übererregbarkeit. Gelegentlich eines Besuches der Ohrenabteilung zeigte das Kind, das einer elektrischen Behandlung unterzogen worden war, die Erscheinungen eines deutlichen Schwindelanfalles. Es war auffallend blaß, zeigte einen müden, ängstlichen Gesichtsausdruck, auffallende Unruhe, Angstgefühl und Unsicherheit beim Gehen. Zugleich fand sich bei seitlicher Blickrichtung (sowohl nach rechts wie nach links) ein deutlicher kleinschlägiger horizontaler Spontannystagmus. Die Untersuchung auf dem Drehstuhle ergab beiderseits gleiche Werte, doch war die Reaktion sowohl bei Drehung nach rechts wie bei Drehung nach links stärker ausgesprochen als bei den ersten Untersuchungen (35 und 37 Sekunden gegenüber 23 und 27 Sekunden). Dabei starker Schwindel. Das Gehör wies gegenüber den ersten Befunden keine Differenz auf.

Überblicken wir die hier mitgeteilten Beobachtungsergebnisse, so können wir zunächst feststellen, daß ein außerordentlich hoher Prozentsatz der untersuchten Kinder neben der erhöhten vasomotorischen Erregbarkeit subjektive und objektive Krankheitserscheinungen von seiten des Gehörorganes darbot.

Die Tatsache der häufigen Koinzidenz von Störungen im Vasomotorenapparate und von Krankheitssymptomen im Bereiche des Gehörorganes stellt uns nun der Frage gegenüber, ob wir es hier mit einem zufälligen Zusammentreffen zu tun haben oder ob wir die erhobenen subjektiven und objektiven Störungen im Hörapparate in ursächliche Beziehung zu den: 
pathologischen Erscheinungen im Zirkulationsapparate bringen dürfen.

Von den funktionellen Störungen, die sich gelegentlich der otiatrischen Untersuchungen nachweisen ließen, kann wohl das Ermüdungsphänomen mit Bestimmtheit als eine Folge der Alteration des Vasomotorenapparates angesehen werden. Es beruht zweifellos auf einer Beeinträchtigung der zerebralen Leistungsfähigkeit infolge sich oft wiederholender und nicht selten länger andauernder angiospastischer Zustände der GehirngefäBe, resp. der mangelhaften Durchblutung derselben. Dafür spricht vor allem das in fast allen Fällen konstatierte Zusammentreffen des Ermüdungsphänomens mit Kopfschmerzen, die gleichfalls aus einer Ischämie der Gehirngefäße resultieren, außerdem aber auch die Beobachtung der ganz eklatanten Wirkung des Allgemeinbefindens a uf die Ermüdungserscheinungen von seiten des Gehörorgans.

Die Erschöpfbarkeit des Nervensystems solcher Kinder zeigt sich in der Zerstreutheit, in dem Versagen, beziehungsweise der Beeinträchtigung der Aufnahmsfähigkeit, in der geistigen Müdigkeit und findet ihren Ausdruck auch in dem Verhalten des Akustikus. Weitere Untersuchungen werden vor allem darauf zu achten haben, ob das Ermüdungsphänomen nicht auch das erste Anzeichen einer organischen Erkrankung im Hörnervengebiete darstellt, was nach diesbezüglichen (mehrjährigen) Beobachtungen Steins an Erwachsenen auBerordentlich wahrscheinlich ist.

Diesubjektiven Gehörsempfindungen, die von jenen Kindern angegeben wurden, bei denen sich objektiv kein pathologischer Befund erheben ließ, dürfen wohl auf Reizzustände im peripheren Neuron des Akustikus zurückgeführt, beziehungsweise mit der Verengerung der das Hörnervengebiet versorgenden Arterien in direkten Zusammenhang gebracht werden. In den Fällen von Erkrankung des inneren Ohres, in denen uiber Ohrgeräusche geklagt wurde, durfte die organische Erkrankung des peripheren Akustikusneurons als pathologisches Substrat der Gehörsempfindungen aufgefaßt werden.

Ohrgeräusche können jedoch bei vasomotorischen Innervationsstörungen auch durch vasodilatatorische Vorgänge zustandekommen und zwar dadurch, daß Gefäßgeräusche, die in den dilatierten Gefäßbahnen entstehen, perzipiert werden, was besonders durch Vermittlung einer pathologischen Verstärkung der Kopfknochenleitung, wie sie in Fällen von Er- 
krankungen des Schallleitungsapparates vorliegt, geschehen kann.

Schließlich können auch die in den sensiblen Nervengebieten des Ohres auftretenden Krankheitserscheinungen zwanglos auf eine Anämisierung der entsprechenden Gefäßbezirke infolge angiospastischer Zustände zurückgeführt werden. Sie sind in Analogie zu bringen mit den von Stein bei Arteriosklerose beschriebenen und als Otalgia angiosclerotica bezeichneten schmerzhaften $\mathrm{Zu}$ ständen, die ihre Ursache in einer durch die Gefäßerkrankung bedingten mangelhaften Durchblutung des sensiblen Nervengebietes des Ohres haben. Den Zusammenhang der schmerzhaften Empfindungen im Bereiche des Ohres mit den Vasomotorenstörungen beleuchtet in sehr eindringlicher Weise der Fall Leidlers, in welchem der Ohrenschmerz auf der Höhe der (objektiv wahrnehmbaren) vasomotorischen, resp. angiospastischen Krankheitserscheinungen auftrat und mit denselben schwand.

Schwieriger ist die Beantwortung der Frage, ob wir vasomotorische Störungen für die Entstehung einer organischen Erkrankung des inneren Ohres verantwortlich machen dürfen.

Wie schon vorerwähnt, sehen Mayer, Sohier Bryant und andere Forscher in den Störungen des Vasomotorenapparates einen bedeutungsvollen ursächlichen Faktor für die Entstehung der Otosklerose, und speziell Mayer bringt die Entwicklung des der Erkrankung zugrundeliegenden Knochenprozesses in direkte Beziehung zu der Zirkulationsstörung, welche durch die Verengerung der zuführenden Gefäße und ungenügende Blutzufuhr die Knochenveränderungen in der Pyramide des Schläfebeines herbeiführt.

Es wäre verlockend, die pathologischen Zirkulationsbedingungen ohne weiteres auch für die Erkrankungen des inneren Ohres als ursächliches Moment zu supponieren, und zwar ganz besonders, wenn wir uns vergegenwärtigen, daß gerade das Hörnervengebiet außerordentliche Ansprüche an die Ernährung, beziehungsweise Blutversorgung stellt. Zur Stütze dieser Annahme ließe sich überdies die unter dem Einflusse arteriosklerotischer Gefäßveränderungen entstehende Erkrankung des Gehörorganes anführen, die unseren klinischen Erfahrungen und anatomischen Befunden zufolge vorwiegend das innere Ohr betrifft und gleichfalls pathologischen Ernährungsverhältnissen durch Gefäßverengerung ihre Entstehung verdankt. 
Zugunsten der Annahme einer aus der vasomotorischen Störung direkt resultierenden Beeinträchtigung des Hörnervengebietes lassen sich zwei unserer Fälle (Nr. 23 und 31) verwerten. Sie zeigten bei gleichzeitigem Bestehen vasomotorischer Störungen im Bereiche der zerebralen Gefäße (Kopfschmerzen, geistige Müdigkeit, auffallende Blässe usw.) die objektiven Erscheinungen der Laesio auris internae, während sich bei allgemeinem Wohlbefinden ein durchaus normaler Funktionsbefund ergab. Die beiden Fälle zeigten somit in eindeutiger Weise, daß eine vorübergehende Störung der Blutzufuhr zum Hörnervengebiete eine Beeinträchtigung seiner Funktion herbeiführen kann, während die Rückkehr normaler Zirkulationsverhältnisse wieder zur normalen funktionellen Leistungsfähigkeit des Gehörorganes führt.

Zur richtigen Beurteilung der übrigen Fälle, in denen sich die Funktionsstörung im inneren Ohre als eine bleibende erwies, erscheint es notwendig, die verschiedenen ätiologischen Momente labyrinthärer Schwerhörigkeit im Kindesalter des näheren zu erörtern.

Die labyrinthäre Schwerhörigkeit im Kindesalter ist in der überwiegenden Mehrzahl der Fälle die Folge von entzündlichen Erkrankungen des Labyrinthes und des Hörnerven, die sich im Verlaufe akuter Infektionskrankheiten eingestellt haben. In solchen Fällen handelt es sich entweder um Labyrinthentzündungen, beziehungsweise -eiterungen, die im Anschlusse an Mittelohreiterungen auftraten und Läsionen der labyrinthären Nervenendstellen nach sich zogen, oder um die Folgeerscheinungen akuter Neuritiden des Hörnerven, die sekundär zu degenerativen Prozessen im Labyrinthe geführt haben.

Wir haben in unseren Fällen sowohl gelegentlich der anamnestischen Erhebungen als auch bei der Untersuchung ganz besonders auf die Möglichkeit eines Zusammenhanges der Innerohrerkrankung mit einem akuten Infekte geachtet.

Für die Annahme einer Erkrankung des inneren Ohres im Anschlusse an eine Eiterung im Mittelohre konnte bei keinem der Kinder ein Anhaltspunkt gewonnen werden. Weder otoskopisch noch durch die Stimmgabelprüfung war irgendein Anzeichen zu eruieren, das auf einen früheren MittelohrprozeB hingewiesen hätte. Die Annahme einer infektiösen Neuritis des Akustikus konnte nicht für alle Fälle mit voller Bestimmtheit abgewiesen werden. Von den anamnestischen Angaben, die wir in den Fällen mit Erkrankungen des inneren Ohres er- 
heben konnten, enthielten die bei sechs Fällen gewonnenen Daten Hinweise auf akute Infektionskrankheiten (in zwei Fällen waren Morbillen, in zwei Fällen war Scarlatina vorausgegangen, in einem Falle enthielt die Krankengeschichte die Angabe, da $\beta$ das Kind vor 7 Jahren an Mumps, vor 6 und 2 Jahren ari Morbillen und vor $1 \frac{1}{2}$ Jahren an Influenza gelitten hätte, und in einem Falle fand sich ein Hinweis auf eine durchgemachte Influenza).

Die Tatsache, daß die Verminderung der Hörschärfe in keinem der in Rede stehenden Fälle in direktem Anschlusse an den akuten Infekt beobachtet worden war, ist für sich allein nicht beweiskräftig genug, da es sich in diesen Fällen um geringe oder mittlere Grade einer Beeinträchtigung des Hörvermögens handelte, die von eîner nicht sorgfältig auf das Kind achtenden Umgebung leicht übersehen werden konnten. Vielleicht lassen sich aber die Krankengeschichten unserer Fälle, denen zufolge die Abnahme des Hörvermögens erst längere Zeit nach der durchgemachten Infektionskrankheit wahrgenommen wurde, in der Weise verwerten, daß wir annehmen, der akute Infekt habe nicht zur Erkrankung des Hörnerven direkt, sondern zunächst zu einer Schädigung des Vasomotorenapparates und erst infolge derselben zur Läsion des Hörnervengebietes geführt.

Des weiteren müssen wir auf der Suche nach der Ätiologie labyrinthärer Schwerhörigkeit im Kindesalter die Möglichkeit ihrer Entstehung als Folge hereditärer Lues diskutieren.

In 14 von unseren 19 Fällen labyrinthärer Erkrankung wurde die Wassermannsche Reaktion ausgefüht, in 6 von ihnen mit positivem Ergebnisse.

Ein positiver Ausfall der Wassermannschen Probe ergab sich in den 3 Fällen mit vollkommener (einseitiger) Taubheit.

In diesen Fällen fanden sich nebst vollständigem Funktionsverluste des akustischen Apparates Veränderungen im statischen Labyrinthe, und zwar konnte bei zweien dieser Fälle eine vollständige Ausschaltung des Vestibularapparates, bei dem dritten eine bedeutende Verminderung seiner Erregbarkeit nachgewiesen werden.

Wiewohl bei den drei Patientinnen an den Zähnen, an den Augen und an den übrigen Organen keine hereditär-syphilitischen Veränderungen nachweisbar waren, so glauben wir doch aus dem positiven Ergebnisse der Wassermannschen Reaktion annehmen zu dürfen, daß es sich in diesen Fällen um 
eine heredoluetische Erkrankung des Nervus octavus und des Ohrlabyrinthes gehandelt habe.

In keinem der drei Fälle hatte sich die Erkrankung des inneren Ohres mit heftigen Erscheinungen kundgegeben. Zwei der Patientinnen waren in das Ohrenambulatorium der Poliklinik gebracht worden mit der Angabe, sie hörten in der letzten Zeit schlechter, eine Tatsache, die aus dem Auftreten, resp. dem Schlechterwerden der Erkrankung auf dem anderen Ohre erklärt werden konnte. Im dritten Falle war die ohrenärztliche Untersuchung des Kindes durch einen akuten Mittelohrkatarrh veranlaßt worden, der das bessere Ohr befallen hatte. Krankheitserscheinungen, welche das Befallen des Vestibularapparates gekennzeichnet hätten (Schwindel, Gleichgewichtsstörungen), fehlten gänzlich. Es erscheint daher überaus wahrscheinlich, daß es sich in den drei Fällen um intrauterin erworbene Labyrinthveränderungen gehandelt habe. In dem einen der drei Fälle wurde eine Jodbehandlung durchgeführt die jedoch ohne jeglichen Erfolg blieb. Der zweite Fall entzog sich schon nach der ersten Untersuchung der weiteren Beobachtung, in dem dritten wurde die antiluetische Behandlung abgelehnt.

In den übrigen drei Fällen mit positivem $W$ assermann, von denen einer eine beiderseitige leichtgradige Erkrankung des inneren Ohres, einer auf dem einen Ohre eine geringgradige, auf dem andern eine mittelgradige Erkrankung des inneren Ohres und der dritte eine beiderseitige mittelgradige Erkrankung des inneren Ohres aufwies, war der statische Apparat beiderseits normal.

Die Frage, ob es sich hier um luetische Veränderungen gehandelt habe, kann nur für einen dieser Fälle (Nr. 32), in welchem sich an den Zähnen und den Augen die für hereditäre Lues charakteristischen Veränderungen zeigten, mit Bestimmtheit in bejahendem Sinne beantwortet werden. In den beiden anderen Fällen muß wohl angesichts des positiven Wassermann die luetische Provenienz der labyrinthären Erkrankung zugegeben werden, doch muß darauf hingewiesen werden, daß die in dem einen dieser Fälle (Nr.9) durchgeführte $\mathrm{Hg}$ - und Jodbehandlung den Ohrbefund in keiner Weise beeinflußte, daß derselbe jedoch bei beiden Kindern Schwankungen aufwies, die der Intensität der vasomotorischen Störungen parallel gingen.

Die Reihe der ätiologischen Momente labyrinthärer Schwerhörigkeit im Kindesalter wäre eine unvollständige, wenn wir nicht noch jener Fälle gedenken würden, in denen die Krank- 
heitserscheinungen des inneren Ohres auf kongenitale Veränderungen im Hörnervenapparate zurückzuführen sind.

Die kongenitale labyrinthäre Schwerhörigkeit ist eine ziemlich seltene Erkrankungsform, für die ein einwandfreier ätiologischer Faktor nicht auffindbar ist. Nach den Erfahrungen Alexanders handelt es sich hier um Fälle, in denen sehr häufig eine hereditäre Belastung vorhanden ist, indem Vater oder Mutter schwerhörig oder taub sind oder kongenitale Taubheit in der Familie, bzw. in der Blutsverwandtschaft beobachtet worden ist. In anderen Fällen fehlt erbliche Belastung, doch sind andere hereditär-degenerative Kennzeichen vorhanden. So sieht man kongenitale labyrinthäre Schwerhörigkeit kombiniert mit geringfügigen Abnormitäten am Gesicht, an den Extremitäten oder am Rumpfe oder es handelt sich um eine allgemeine nervöse Belastung und das Vorkommen von Nerven- und Gehirnerkrankungen in der Aszendenz oder endlich um hereditäre Lues.

Die anatomischen Veränderungen bei der kongenitalen labyrinthären Schwerhörigkeit bestehen nach Alexander in einer kongenitalen Hypoplasie des Ganglion spirale und des peripheren Anteiles der Schneckennerven. Die Kerne, Wurzeln und die zentrale Verzweigung des Kochlearis erweisen sich dagegen als vollständig intakt. Das Cortische Organ und die Stria vascularis ergeben, wie bei der labyrinthären Schwerhörigkeit des Erwachsenen, verschiedene Formen und Grade der degenerativen Atrophie.

Bezüglich des Krankheitsbildes erwähnt Alexander vor allem, daß bei einseitigem Auftreten des Prozesses und bei Fehlen von Erscheinungen von seiten des Vestibularis die labyrinthäre Schwerhörigkeit jahrelang symptomlos bestehen kann, bis sie endlich zufällig oder infolge einer Erkrankung des gesunden Ohres entdeckt wird.

In die gleiche Gruppe von Erkrankungen gehört auch die zuerst von Politzer beschriebene progressive labyrint häre Schwerhörigkeit des jugendlichen Alters. Es handelt sich hier um Fälle, bei welchen am Ende des zweiten Dezenniums eine rasch zunehmende beiderseitige Schwerhörigkeit eintritt, die nach kürzerer oder längerer Zeit zu hochgradiger Herabsetzung des Hörvermögens oder völliger Taubheit führt. Die Kenntnis dieser Zustände ist durch Manasse in sehr wertvoller Weise gefördert worden. Politzer erblickt das anatomische Substrat dieser Erkrankungen in einer idiopathischen Atrophie des Akustikus. Nach Alexander dürfte ein Teil dieser Fälle auf 
der Basis einer kongenitalen Labyrinthschwerhörigkeit zur Entwicklung gelangen. In ätiologischer Beziehung werden erbilche Belastung mit Ohr- oder Geisteskrankheiten oder anderen kongenitalen Bildungsanomalien und hereditäre Lues hervorgehoben.

Erkrankungen des inneren Ohres, die in die hier vorgeführten Gruppen von pathologischen Prozessen des Labyrinthes gehören, können, wie schon erwähnt, leicht übersehen werden, wenn sie - selbst bei hochgradigen Veränderungen im Hörnervenapparate - einseitig auftreten; dasselbe gilt aber auch von beiderseitigen Erkrankungen, sofern dieselben nicht allzuschweren Grades sind.

Bevor wir demnach im Kindesalter nachweisbare Erkrankungen des inneren Ohres mangels anderer ätiologiseher Momente auf vasomotorische Störungen zurückführen dürfen, müssen wir auch die Möglichkeit einer etwa vorliegenden kongenitalen Erkrankung des inneren Ohres zur Diskussion stellen.

Anhaltspunkte für die Annahme eines kongenitalen Prozesses im Hörnervenapparate können aus den anamnestischen Erhebungen, soweit sie sich auf den Patienten selbst und auf seine Familie, resp. Aszendenz beziehen, einerseits und aus den Ergebnissen der klinischen Untersuchung andererseits gewonnen werden.

Im nachfolgenden seien sechs Fälle unseres Beobachtungsmaterials angeführt, in denen wir auf Grund der Anamnese und Untersuchung Anhaltspunkte von großer Prägnanz für die Annahme einer Entwicklung des Ohrenleidens auf dem Boden einer kongenitalen Disposition gewinnen konnten.

T. (Fall Nr. 10). 11 jähriger Bildhauergehilfenssohn.

Ohrbefund: Beiderseitige leichtgradige Erkrankung des inneren Ohres.

Vater Epileptiker, hört seit vielen Jahren schlecht. Ein Bruder des Vaters, der an Tuberkulose litt, ist an Gehirnhautentziindung gestorben. Großvater väterlicherseits an Tuberkulose gestorben. Großvater mütterlicherseits, immer schwächlich und kränklich, ist plötzlich gestorben, Großmutter mütterlicherseits nierenkrank und herzleidend. Eine Schwester der Mutter mit 41 Jahren an Lungentuberkulose gestorben (litt an Strabismus). Eine Schwester des Patienten leidet an Strabismus convergens, eine zweite an Lungenspitzenkatarrh.

Patient hat spät gehen gelernt, leidet oft an Kopfschmerzen, lernt schlecht. 
Allgemeiner Status*): Rachitische Veränderungen am Schädel. Abnorme Zahnstellung. Drüsenschwellung am Halse. Adenoide Vegetationen. Große Tonsillen. Hoher Gaumen. Rachenreflex und Kornealreflexe fehlen, Sehnenreflexe stark gesteigert. Dermographismus. Labiles Herz. Strabismus convergens.

II. (Fall Nr. 18). 9jähriger Wirkerssohn.

Ohrbefund: Rechtes Ohr normal. Links : leichtgradige Erkrankung des inneren Ohres.

Großvater mütterlicherseits an Herzmuskelentartung, Großmutter an Ca. uteri gestorben. Mutter leidet an Morbus Basedowii. Ein Bruder des Patienten leidet an Chorea.

Patient lernt schlecht, sein Gedächtnis ist mangelhaft, sein Schlaf unruhig.

Allgemeiner Status: Asthenischer Habitus. Naevus pigmentosus in der rechten Nasolabialfalte. Sehr kariöse Zähne. Hyperplasie des lymphatischen Rachenringes. Rachenreflex und Kornealreflexe fehlen, Sehnenreflexe gesteigert. Dermographismus. Respiratorische Irregularität des Pulses. Erbensches Pulsphänomen. Systolisches Geräusch am Herzen.

III. (Fall Nr. 27.) 13jähriger Gärtnergehilfenssohn.

Ohrbefund: Beiderseitige leichtgradige Erkrankung des inneren Ohres.

Eine Schwester des Patienten ist augenkrank (Astigmatismus myopicus, Strabismus divergens alternans).

Patient leidet an Kopfschmerzen und häufig auftretendem Surren in den Ohren. Gehör schwankend.

Allgemeiner Status : Kornealreflexe und Rachenreflex fehlen, Sehnen- und Bauchdeckenreflexe gesteigert. Leichte Vergrößerung der Schilddrüse. Hyperplasie des lymphatischen Rachenringes. Dermoid im rechten äußeren Augenwinkel. Labiles Herz (Erbe n sches Pulsphänomen, As ch ner scher Bulbusdruckreflex respiratorische Irregularität). Links offener äußerer Leistenring.

IV. (Fall Nr. 33.) 11 jähriger Buchhalterssohn.

Ohrbefund: Rechts geringgradige, links mittelgradige Erkrankung des inneren Ohres.

Eine Schwester schwerhörig. Übrige Familienanamnese belanglos.

Allgemeiner Status: Asymmetrie des Gesichtes. Chvostek +, Thymusdämpfung (?), Rachenreflex und Kornealreflexe herabgesetzt. Sehnen- und Bauchdecken-

*) Die bei den nachfolgenden Fällen angeführten Befunde danken wir den für uns gütigst durchgeführten Untersuchungen des Herrn Dr. Julius Bauer, Assistenten der Abteilung Prof. Mannaberg. 
reflexe gesteigert. Cor sehr labil, systolisches Geräusch, schwach, über der Pulmonalis. Dermographismus. Crines pubis auffallend stark entwickelt.

V. (Fall Nr. 34.) 13jähriger Kaufmannssohn.

Ohrbefund: Beiderseitige mittelgradige Erkrankung des inneren Ohres.

Großvater väterlicherseits, 9 Jahre gelähmt, erlag einem. Schlaganfalle, Großmutter mütterlicherseits im Alter von 42 Jahren an Magenkrebs, Großvater mütterlicherseits an einem Herzleiden gestorben. Mutter nierenkrank, hört seit einem Jahre schlecht (labyrinthäre Schwerhörigkeit). Eine Schwester und ein Bruder des Vaters an Tuberkulose, ein Bruder des Patienten an Gehirnhautentzündung gestorben. Eine Schwester des Patienten leidet an Tuberkulose.

Patient schläft schlecht, leidet oft an Bauchschmerzen und Diarrhöen. Zeitweise Summen in den Ohren.

Allgemeiner Status: Asthenischer Habitus. Apicitis bilateralis. Drüsensehwellung am Halse. Spitzbogengaumen. Hyperplasie des lymphatischen Rachenringes. Rachenreflex und Kornealreflexe fehlen, Periost- und Sehnenreflexe gesteigert. Dermographismus. Labiles Herz.

VI. (Fall 35.) 13jähriger Dienstmannssohn.

Links mittelgradige, rechts leichtgradige Erkrankung des inneren Ohres.

Großvater mütterlicherseits: Rheumatismus, Herzleiden. Großmutter mütterlicherseits: leberleidend. Mutter leidet an Gallensteinen und an leichtgradiger labyrinthärer Schwerhörigkeit, Vater ist Alkoholiker. Ein Bruder der Mutter: Epileptiker, ein Bruder des Vaters schwachsinnig. Eine Schwester des Patienten (17 Jahre alt): Struma parenchymatosa.

Allgemeiner Status: Synophris. Angewachsene Ohrläppchen. Hoher Gaumen. Rachenreflex und Kornealreflexe fehlen, Sehnen- und Bauchdeckenreflexe stark gesteigert. Rigide Arteriae rad. et temporal. Dermographismus sehr intensiv. Respir. Irregularität. Er bensches Pulsphänomen. Scap. scaphoid. Crines pub. weiblich, gut entwickelt. Auffallend unproportionierter, enormer Penis.

Die hier mitgeteilten Krankengeschichten lassen in den angeführten Details in einwandfreier Weise die hereditärdegenerative Anlage der Untersuchten erkennen. Sie zeigt sich einerseits in der großen Zahl degenerativer Stigmen, die bei einem und demselben Individuum rorgefunden wurden und andererseits in den Details der Familienanamnese, die eine 
kleinere oder größere Reihe von in degenerativem Boden wurzelnden Erkrankungen aufweist.

Eines der Symptome degenerativer Veranlagung ist nun auch die vasomotorische Übererregbarkeit, die wir in allen unseren Fällen in eklatanter Weise feststellen konnten.

Die Anomalien der Vasomotoren und die gleichzeitig nachweisbare Labilität der Herzaktion bilden ein wichtiges Kriterium zur Feststellung der abnormen Körperverfassung, und ihre Verwertung als Bindeglied in der Kette der nachgewiesenen degenerativen Stigmen führt zum richtigen Verständnisse der hier vorliegenden Beziehungen.

J. Bau er und C. Stein haben bei 26 Fällen von Otosklerose die Häufung angeborener Entartungszeichen und ihr konstantes Vorkommen festgestellt und sehen in der hereditärdegenerativen Anlage solcher Patienten die Erklärung für die abnorme Reaktion des Organismus auf irgendwelche Reize. Das gilt speziell auch für das Gehörorgan, für das sie eine in der Keimesanlage begründete Minderwertigkeit supponieren.

Das in allen untersuchten Fällen von Otosklerose nachzuweisende Symptom degenerativer Veranlagung ist die Labilität der Blutversorgung, welche ihre Ursache in der immer festzustellenden Übererregbarkeit der Vasomotoren und in der Labilität der Herzaktion hat. Die Ansicht Mayers, daß die anatomischen Veränderungen der Otosklerose durch Störungen der Vasomotoren veranlaßt werden, erscheint demnach gewiß zutreffend, doch muß die stets zu konstatierende hereditär-degenerative Veranlagung als Conditio sine qua non zugrunde gelegt werden.

Dieselben Beziehungen ergeben sich nun auch für die hier mitgeteilten 6 Fälle hinsichtlich des inneren Ohres. Auch hier bietet das konstitutionelle Moment eine Handhabe für das Verständnis der Pathogenese der Ohrerkrankung. Die wichtigste Voraussetzung liegt in der hereditär-degenerativen Anlage des Individuums und in der Minderwertigkeit des Gehörorganes, das von vasomotorischen Störungen ganz besonders ungünstig beeinflußt wird und ihren schädigenden Wirkungen schon frühzeitig unterliegt. So erscheint es leicht verständlich, daß alle Momente, welche den Vasomotorenapparat schädigen (psychische Erregungen, Traumen, Bakterientoxine, infektiöse Einflüsse), die Entwicklung des in der Keimesanlage begründeten Leidens auslösen, beziehungsweise fördern. So können wir auch verstehen, daß sich die ersten Anzeichen des Ohrenleidens in der Zeit der Pubertät offenbaren. Es handelt 
sich eben um die Störung des Blutdrüsengleichgewichtes, welche die Vasomotoren in wesentlichem Maße tangiert und die Progredienz des bis dahin kaum erkennbaren Leidens herbeiführt.

Kommen wir nun auf Grundlage der vorliegenden Erörterungen, in denen wir die Ätiologie der Erkrankung des inneren Ohres in 19 Fällen unseres Beobachtungsmateriales zur Diskussion gebracht haben, auf die Frage zurück, ob vasomotorische Störungen eine organische Schädigung des Hörnervenapparates nach sich ziehen können, so ergibt sich unserer Ansicht nach folgendes:

Vasomotorische Störungen können durch vasokonstriktorische Vorgänge und die sich hieraus ergebende Alteration der Blutzufuhr den Hörnerven und seine labyrinthären Endapparate in mehr oder weniger ausgesprochener Weise funktionell schädigen. Die Schädigung findet ihren Ausdruck in objektiv feststellbaren Funktionsausfallserscheinungen, die bei geringer Dauer der Zirkulationsstörung nur vorübergehend auftreten und mit Herstellung normaler Zirkulationsverhältnisse wieder vollständig verschwinden können. Ihre $\mathrm{Zu}$ sammengehörigkeit mit den vasomotorischen Krankheitserscheinungen dokumentiert sich in unzweifelhafter Weise in dem Parallelismus der Krankheitssymptome von seiten des Zirkulationsapparates und jener von seiten des inneren Ohres.

Bei häufiger Wiederholung solcher die Ernährung des inneren Ohres alterierenden vasokonstriktorischen Attacken oder bei langer Dauer der vasomotorischen Störungen kann es zweifelsohne zur Entwicklung irreparabler organischer Veränderungen im inneren Ohre kommen.

Unter den Faktoren, welche den Vasomotorenapparat in ganz besonderem Maße zu schädigen vermögen und auf diesem Wege die Entstehung innerer Ohrerkrankungen herbeizuführen imstande sind, nehmen toxische und infektiöse Einflüsse eine bedeutsame Stellung ein.

Zweifellos gibt es toxische, infektiöse (und wohl auch traumatische) Erkrankungen des inneren Ohres, bei denen sich Krankheitserscheinungen, welche durch direkte Schädigung des Hörnervenapparates hervorgerufen werden, und solche, die vasomotorischen Charakters sind, summieren. Die Schwankungen, die sich in den objektiven und subjektiven Krankheitserscheinungen von seiten des Gehörorganes zeigen, dürfen wohl ausschließlich auf Rechnung der vasomotorischen Komponente der Krankheitsbilder gesetzt werden.

Die Behauptung, daß nach akuten Infektionskrankheiten 
oder toxischen Einflüssen aufgetretene Krankheitserscheinungen von seiten des inneren Ohres vasomotorischen Ursprungs sind, darf wohl dort aufgestellt werden, wo sich die Erscheinungen der Funktionsstörung im Hörnervenapparate erst längere Zeit nach Einwirkung der infektiösen, beziehungsweise toxischen Schädlichkeit zeigen, vor allem aber in jenen Fällen, in denen anfänglich temporäre Störungen im Hörnervengebiete nach längerer Zeit zu dauernden geworden sind.

Daß auch chronische Infektionskrankheiten, wie auch besonders die Lues den Hörnerven und das Labyrinth nicht nur direkt, sondern auch auf dem Wege des Vasomotorenapparates zu schädigen vermögen; darf wohl für einzelne Fälle zugegeben werden.

Als klinisch besonders bedeutsam muß die Tatsache bezeichnet werden, daß die vasomotorische Übererregbarkeit in manchen Fällen eine Teilerscheinung in dem vielgestaltigen Bilde der hereditär-degenerativen Veranlagung bildet. Tangiert die Labilität der Vasomotoren ein von Haus aus minderwertiges Gehörorgan, wie es in solchen Fällen nicht selten der Fall ist, so erscheint es leicht verständlich, daß sich die ungünstigen Wirkungen der labilen Blutversorgung auf das innere Ohr frühzeitiger und in weitaus höherem Grade als unter anderen Umständen bemerkbar machen.

In knapper Präzisierung unseres Standpunktes in der Frage über den Einfluß vasomotorischer Störungen im Kindesalter auf das Gehörorgan formulieren wir unsere Ansichten an der Hand klinischer Beobachtungen in folgenden Schlußsätzen:

Die vasomotorische UUbererregbarkeit im Kindesalter ruft neben Störungen in verschiedenen Organen überaus häufig auch solche im Gehörorgan hervor.

Die Krankheitserscheinungen von seiten des Ohres resultieren zweifellos aus der Beeinträchtigung der Zirkulation im Gehörorgane und äußern sich, als Folge einer Anämisierung des betreffenden Gebietes, in der überwiegenden Mehrzahl der Fälle im Bereiche des Hörnervenapparates.

Sie finden ihren Ausdruck in subjektiven Beschwerden und in objektiven Krankheitserscheinungen von seiten des Ohres.

$\mathrm{Zu}$ den subjektiven Beschwerden zählen: subjektive Ohrgeräusche und schmerzhafte Sensationen, zu den 
objektiven Symptomen: Ermüdungserscheinungen von seiten des Akustikus, Beeinträchtigung der Funktion des Kochlearapparates, in vereinzelten Fällen des Vestibularapparates.

Zur Beweisführung für den direkten Zusammenhang der vasomotorischen Störungen mit jenen im Gehörorgane können zwei Momente herangezogen werden: 1. der Umstand, daß die Krankheitserscheinungen von seiten des Ohres fast immer im Rahmen des charakteristischen Krankheitsbildes zerebraler vasomotorischer Störungen auftreten (Kopfschmerzen, Schwindel, geistige Ermüdbarkeit usw.), und 2. die Tatsache, daß die Beeinträchtigung der funktionellen Leistungen des inneren Ohres in überzeugender Weise der Intensität der vasomotorischen Störungen parallel geht.

Die Frage, ob vasomotorische Störungen auch organische Veränderungen im Hörnervengebiete veranlassen können, ist unbedingt zu bejahen, und zwar ganz besonders für Fälle, in denen das Gehörorgan auf der Grundlage einer allgemeinen degenerativen Veranlagung von Haus aus minderwertig ist. In solchen Fällen können alle Faktoren, welche eine abnorme Funktion der Vasomotoren auszulösen vermögen (Infektionskrankheiten, psychische und physische Traumen, Störungen des Blutdrüsengleichgewichts), die Entwicklung der Erkrankung des inneren Ohres herbeiführen oder zum mindesten beschleunigen.

Mit Rücksicht auf den Mangel größerer Erfahrungen in der Behandlung vasomotorischer Störungen des Gehörorganes möchten wir die therapeutische Seite des vorliegenden Themas an dieser Stelle nicht näher erörtern, sondern nur auf die hier in Betracht kommenden wichtigsten Momente hinweisen. Die Aussichten der Behandlung vasomotorischer Störungen auf die von ihnen im Ohre hervorgerufenen Anomalien könnèn unserer Ansicht nach nur nach jahrelang fortgesetzten Beobachtungen eingehender zur Sprache gebracht werden.

Die Hauptaufgaben der Behandlung sind: Ausschaltung aller die Vasomotoren schädigenden Faktoren, Verordnung entsprechend gewählter roborierender Diät, richtiges Ausma $B$ der Anforderungen an die körperliche und geistige Leistungsfähigkeit des Kindes und ganz besonders auch sorgfältige Kontrolle aller Schuleinflüsse auf sein Nervensystem.

Besonderen Nachdruck möchten wir auf die Tat- 
sache legen, daß durch sorgfältige Beobachtungen von krankhaften Störungen im Gehörorgan als Folgeerscheinungen vasomotorischer Übererregbarkeit im Kindesalter schon frühzeitig ein Leiden konstatiert werden kann, dessen weitere Entwicklung im fortschreitenden Alter unter allen Umständen befürchtet werden muß. Einem zielbewußten Eingreifen zu richtiger Zeit wird es vielleicht doch gelingen, der Entwicklung der krankhaften Vorgänge vorzubeugen oder wenigstens ihrer raschen Progredienz Einhalt zu tun.

Krankengeschichten der untersuchten Fälle.

I. Otto K., 9 jähriger Fabriksarbeiterssohn aus Berndorf. Sehr blaß, mager, von unruhigem Wesen, sehr ängstlich und schüchtern. Nach Angabe der Mutter sehr nervös, leicht ermüdend; bleibt in der Schule zuriuck. Kopfschmerzen, Appetitlosigkeit, häufig (besonders nach Gemütserregungen) Harndrang.

Ohrbefund: Trommelfell beiderseits normal. Uhr rechts $30 \mathrm{~cm}$, links $10 \mathrm{~cm}$, Flüstersprache rechts $10 \mathrm{~m}$, links $5 \mathrm{~m}$. Laute Sprache beiderseits $+10 \mathrm{~m}$. Weber nach rechts, Rinne beiderseits positiv, Sehwabach rechts 4-5" links $10^{\text {" }}$ verkürzt. $c^{4}$ links 20 Sek. verkürzt. Ermüdungsphänomen beiderseits. Statischer Apparat normal.

Diagnose: Rechts geringgradige, links mittelgradige Erkrankung des inneren Ohres.

II. Paul Josef E. 7 Jahre. Vor 2 Jahren Morbillen. Seither Erscheinungen allgemeiner Nervosität und Blutarmut: Schlechtes Aussehen, Appetitlosigkeit, leichte Ermüdbarkeit, grundloser Stimmungswechsel, Reizbarkeit, Kopfschmerzen, mitunter Schwindelgefühl, Häufig auftretende, besonders zur Zeit der Kopfschmerzen fühlbar werdende stechende Schmerzen in der Schläfen- und Warzenfortsatzgegend. Die interne Untersuchung ergibt neben typischen vasomotorischen Erscheinungen eine sehr deutlich ansgesprochene Labilität des Herzens.

Ohrbefund: Trommelfell beiderseits normal. Uhr: rechts $40 \mathrm{~cm}$, links $20 \mathrm{~cm}$, Flüstersprache rechts $10 \mathrm{~m}$, links $6-7 \mathrm{~m}$. Weber unbestimmt, Rinne beiderseits positiv, Schwabach rechts normal, links ca. 20 Sek. verkürzt. $e^{4}$ links leicht verkürzt. Vestibularapparat normal.

Diagnose: Rechts geringgradige, links mittelgradige Erkrankung des inneren Ohres.

III. Robert B., 13 jähriger Kaufmannssohn. Vor 7 Jahren Mumps, vor 6 und 2 Jahren Morbillen, vor einem halben Jahre Influenza. Klagt über hänfiges Schwindelgefühl, Kopfschmerzen, zeitweise auftretendes Gefühl von Kurzatmigkeit, in der letzten Zeit mitunter bald im rechten, bald im linken Ohre auftretende Schmerzen. Mager, hoch aufgeschossen, Gesicht sehr blaß mit grünlichem Kolorit. Von unruhigem Wesen; nervöses Husten und Räuspern. Sehr stark ausgesprochene vasomotorische Erscheinungen, rigide Art. tempor. und rad.

Otoskopischer Befund normal. Hörvermögen: Uhr rechts $2 \mathrm{~cm}$, links $10 \mathrm{~cm}$. Flüstersprache rechts $11 / 2 \mathrm{~m}$, links $2-21 / 2 \mathrm{~m}$. Weber im Kopf. Rinne beiderseits positiv. Schwabach beiderseits (r. mehr als 1.) verkürzt. $c^{\mathfrak{d}}$ rechts stark, links in geringem Grade verkürzt. Vestibularapparat normal. 
Diagnose: Rechts hochgradige, links mittelgradige Erkrankung des inneren Ohres.

IV. Josef L. 7 Jahre. Keine Beschwerde von seiten des Gehörorganes. Normales Gehör. Normaler Funktionsbefund.

V. Marie U. 11 jährige Geschäftsdienerstochter. Keine Beschwerden von seiten des Ohres. Normales Gehör. Eklatantes Ermüdungsphänomen beiderseits, im übrigen normaler Funktionsbefund.

VI. Walter K. 12 jähriger Fabriksbeamtenssohn aus Berndorf. Häufig Kopfsehmerzen, Schwindel, Magenschuerzen, oît obstipiert.

Ohrbefund: Trommelfelle beiderseits normal. Uhr rechts $70 \mathrm{~cm}$, links $40 \mathrm{~cm}$, Flüstersprache rechts $10 \mathrm{~m}$, links ca. $8 \mathrm{~m}$. Weber nach rechts. Rinne beiderseits positiv. Schwabach rechts normal, links 5 Sek. verkürzt. Vestibularapparat normal.

Diagnose: Rechtes Ohr normal, links geringgradige Erkrankung des inneren Ohres.

VII. Marie P. 10 Jahre. Klagt über Schmerzen, die bald im rechten Ohre, bald im linken auftreten und 10--15 Minuten, manchmal bis zur Dauer einer halben Stunde anhalten. Patientin hat dann gewöhnlich das Gefüh1, ,als wäre etwas wund im Ohre". Normales Gehör. Eklatantes Ermüdungsphänomen beiderseits. Vestibularapparat normal.

VIII. Franz Sch. 5 Jahre. Seit einem Jahre ohne Ursache schlechtes Aussehen, Appetitlosigkeit, unruhiger Schlaf. Seit gleicher Zeit merkt die Umgebung des Kindes, daß dasselbe schlechter höre.

Ohrbefund: Trommelfell beiderseits normal. Uhr: beiderseits al concham. Fliistersprache rechts $1 \mathrm{~m}$, links $2 \mathrm{~m}$. Laute Sprache beiderm seits $4 \mathrm{~m}$. Weber i. K. Rinne positiv. Schwabach beiderseits stark verkürzt. $c^{4}$ beiderseits verkürzt. Vestibularapparat normal.

Diagnose: Beiderseitige hochgradige Erkrankung des inneren Ohres.

IX. Alfred W. 9 jähriger Lehrerssohn. Kopfschmerzen, Appetitlosigkeit, leichte Ermüabarkeit, Bewegungsstörungen (allgemeine Unruhe, Zupfen, Zappeln usw.). Während der Kopfschmerzen Zerstreut. heit, große Müdigkeit, Klingen oder Summen in den Ohren. Wassermann positiv.

Ohrbefund: (bei wiederholten Untersuchungen differierend): Uhr beiderseits $30-40 \mathrm{~cm}$, Flüstersprache beiderseits $6-10 \mathrm{~m}$. Weber unbestimmt. Rinne beiderseits positiv. Schwabach beiderseits ca. 10 Sek. verkürzt. 0 rechts 5 , links 8 Sek. verkürzt. Beiderseits sehr deutliches Ermüdungsphänomen.

Diagnose: Beiderseitige geringgradige Erkrankung des inneren Ohres mit starken, dem Allgemeinbefinden parallel gehenden Schwankungen im. Funktionsbefunde des aku * stischen Apparates.

Eine Hg - und Jodkur bewirkte keine Änderung im Funktionsbefunde.

X. Johann H. 11 jähriger Bildhauergehilfenssohn. Vater Epileptiker, hört seit vielen Jahren schlecht. Großvater mütterlicherseits immer schwächlich und kränklich, ist plötzlich gestorben. Großmutter mütterlicherseits nierenkrank und herzleidend. Eine Schwester der Mutter mit 41 Jahren an Lungentuberkulose gestorben (litt an Strabismus). Ein Bruder des Vaters, der an Tuberkulose litt, ist an Gehirnhautentzündung gestorben. GroBvater väterlicherseits an Tuberkulose gestorben. Eine Schwester des Patienten leidet an Strabismus convergens, eine zweite an Lungenspitzenkatarrh. Patient hat spät gehen gelernt, klagt oft über Kopfschmerzen, lernt immer schlecht. Leidet zeitweise an 
Ohrensausen, hört mitunter schlecht. Manchmal Schmerzen in den Ohren.

Allgemeiner Status: Rachitische Veränderungen am Schädel. Abnorme Zahnstellung. Drüsenschwellung am Halse. Adenoide Vegetationen. Große Tonsillen. Hoher Gaumen. Rachenreflex und Kornealreflexe fehlen, Sehnenreflexe stark gesteigert. Dermographismus. Labiles Herz. Strabismus convergens.

Ohrbefund: Trommelfell beiderseits normal. Uhr: rechts $50 \mathrm{~cm}$, links $40 \mathrm{~cm}$. Flüstersprache beiderseits $10 \mathrm{~m}$. Weber im Kopf, Rinne beiderseits positiv, Schwabach beiderseits ca. 10 Sek. verkürzt. $\mathrm{c}^{4}$ beiderseits ca. 6 Sekunden verkürzt, $C$ rechts normal, links 5 Sek. verkürzt.

Diagnose: Beiderseitige geringgradige Erkrankung des inneren Ohres.

XI. Melanie Sch. 8 jährige Kaufmannstochter. Sehr nervöses, debiles Kind. Schläft unruhig, ist sehr zerstreut, klagt über häufig wiederkehrende, oft stundenlang andauernde Kopfschmerzen, manchmal über Schwindel. Schläft mit offenem Munde, schnarcht in der Nacht. I.eidet oft an Schnupfen und klagt während desselben über quälendes Ohren. sausen. Zeitweise Schmerzen in den Ohren. acuta.

Rhinoskopischer Befund: Adenoide Vegetationen, Rhinitis sub-

Obrbefund: Beiderseitiger akuter Mittelobrkatarrh mit mäßiger Herabsetzung der Hörschärfe. Inneres Ohr normal. Nach Luft dusche normale Hörschärfe. Nach Fntfernung der adenoiden Vegetationen schwinden die Krankheitserscheinungen von seiten des Gehörorganes vollständig.

XIT. Raimund Pr. 13jähriger Musikenssohn. Deutliche mäßiggradige Erscheinungen vasomotorischer Innervationslabilität.

\section{Ohrbefund: Vollständig normal.}

XIII. Em ma E. 6 jährige Dienerstochter. Sehr zartes, auBerordentlich blasses, müde aussehendes Kind. Leidet seit einem halben Jahre an Kopfschmerzen, die anfangs nur nach längeren Zeiträumen auftraten, seit 3 Monaten aber täglich wiederkehren und sich oft auch nachts einstellen (Schläfe- und Stirngegend). Sehr oft werden die Kopfschmerzen von Ohrenstechen begleitet. Die Mutter erzählt, das Kind schreie oft plötzlich auf und greife sich mit den Händen an die Ohren oder hinter dieselben.

Ohrbefund: Normale Trommelfelle. Hörschärfe beiderseits normal. Beiderseits Ermüdungsphänomen.

XIV. Heinrich J. $12 \frac{1}{2}$ Jabre. Häufig wiederkehrende Kopfschmerzen, Trommelfell beiderseits normal. Beiderseits eklatantes Ermüdungsphänomen, sonst normaler Ohrbefund.

XV. Richard P. $9 \frac{1}{2}$ jähriger Hilfsarbeiterssohn. Vor 3 Jahren beiderseitige $O$ titis von mehrwöchentlicher Dauer. Vor einigen Wochen erhielt Patient einen Schlag auf das rechte Ohr. Seither konstantes Pfeifen im rechten Ohre. Häufig Kopfschmerzen.

Ohrbefund: Trommelfelle beiderseits normal. Uhr beiderseits normal. Flüstersprache beiderseits $+10 \mathrm{~m}$. Kopfknochenleitung normal. $c^{4}$ beiderseits gleich gut gehört, $\mathrm{C}$ links etwas verkürzt. Eklatantes Ermüdungsphänomen beiderseits.

XVI. Franz H. 13 jähriger Kupferschmiedgehilfenssohn. Typische vasomotorische Krankheitserscheinungen mit vorwiegender Lokalisation im Verdauungs- und Bewegungsapparate.

Ohrbefund: Vollständig normal.

XVII. Marie P. 12 jährige Kutscherstochter. Deutliche mäBiggradige vasomotorische Krankheitserscheinungen. Adenoide Vegetationen. Hypertrophia tonsillarum. 
Ohrbefund: Beiderseits akute katarrhalische Erscheinungen im Mittelohre mit mäßiger Herabsetzung des Hörvermögens Beiderseits deutliches Ermüdungsphänomen.

Nach Entfernung der adenoiden Vegetationen und hypertrophischen Tonsillen und einigen Lufteinblasungen normales Gehör. Das Ermüdungsphänomen zeigte sich auch weiterhin in unverändertem Grade.

XVIII. Friedrich St. 9 jähriger Wirkerssohn. Großvater mütterlicherseits an Herzmuskelentartung, Großmutter mütterlicherseits an Carcinoma utexi gestorben. Mutter: Morbus Basedowii. Ein Bruder des Patienten: Chorea. Patient machte vor 3 Jahren Morbillen durch. Zeitweise auftretende, vom Gehörgange aus über das Ohr bis in die Schläfe- oder Scheitelgegend, manchmal in die Hinterhauptsgegend ausstrahlende reißende Schmerzen auf der linken Seite.

Allgemeiner Status: Asthenischer Habitus. Naevus pigmentosus in der rechten Nasolabialfalte. Sehr kariöse Zähne. Hyperplasie des lymphatischen Rachenringes. Rachenreflex und Kornealreflexe fehlen, Sehnenreflexe gesteigert. Dermographismus. Respiratorische Irregularität des Pulses. Erbensches Pulsphänomen. Systolisches Geräusch am Herzen.

Ohrbefund: Trommelfell beiderseits normal. Uhr rechts $75 \mathrm{~cm}$, links $30-40 \mathrm{~cm}$. Flüsters prache rechts normal, links $6-8 \mathrm{~m}$. c rechts normal, links $15-20$ Sek, verkürzt. Schwabach links verkürzt. Sehr deutliches Ermüdungsphänomen. Starke Schwankungen im Funktionsbefunde: bei gutem Allgemeinbefinden besseres, bei schlechtem Allgemeinbefinden schlechteres Hörvermögen. Vestibularapparat normal.

Diagnose: Geringgradige Erkiankung des linken inneren Ohres.

XrX. Richard S. 8 Jahre. R.: Chronische Mittelohreiterung. I.: normaler otoskopischer Befund. Weber nach rechts. Rinne rechts negativ, links positiv. Schwabach rechts verlängert. Gehör rechts vermindert (Uhr $5 \mathrm{~cm}$, Flüstersprache $4 \mathrm{~m}$, laute Sprache $8 \mathrm{~m}$ ), links normal.

XX. Franziska Pr. 8 jährige Heizerstochter. Leidet an zeitweise auftretendem Schwindel und gleichzeitig bestehender Utbelkeit. Mitunter subjektive Ohrgeräusche (Summen einer Biene oder Sausen wie der Wind).

Hörvermögen normal. Funktionsbefund normal.

XXI. Ella F, 8 Jahre. Kopfschmerzen, leichte Ermüdbarkeit, Appetitlosigkeit, Verstopfung. Mitunter Schwindel und Klingen in den Ohren.

Normales Gehör. Beiderseits Ermüdungsphänomen,im übrigen normaler Funktionsbefund.

XXII. Rudolf Kr. 9 Jahre. Anfälle von Kopfschmerzen mit Schmerzen in beiden Ohren (besonders nach geistiger oder körperlicher Ermüdung). Appetitlosigkeit. Häufig Bauchschmerzen. Bewegungsstörungen (Tic, allgemeine Tinruhe).

Normales Gehör. Beiderseits deutliches Ermïdungsphänomen, sonst normaler Funktionsbefund.

XXIII. Walter En. 9 jähriger Lehrerssohn. Seit einer vor einem halben Jahre durchgemachten Influenza zeigen sich bei dem Knaben schon nach geringfügigen geistigen und körperlichen Anstrengungen Ermüdungserscheinungen. Häufig Appetitlosigkeit und Obstipation. Zeitweise Harndrang. Kopfschmerzen, Schwindel, mitunter Summen oder Klingen bald im rechten, bald im linken Ohre. In den letzten Wochen machte sich bei dem Knaben eine Abnahme der Hörschärfe geltend, welche die Eltern veranlaßte, ihn ohrenärztlich untersuchen zu lassen.

Trommelfell beiderseits normal. Der Funktionsbefund zeigte sich 
gelegentlich mehrfach wiederholter Untersuchungen außerordentlich variabel. An Tagen, an denen das Allgemeinbefinden des Patienten ein gutes war, fand sich ein normales Gehör und vollständig normaler Funktionsbefund oder ein- bis zweimal zu konstatierendes Ermüdungsphänomen. Bei schlechtem Allgemeinbefinden (an solchen Tagen bestanden außerordentliche Blässe, fast grünliches Kolorit, dunkel umränderte Augen, kalte, bläuliche Hände, große Mattigkeit) ergaben sich alle Zeichen der Erkrankung des inneren Ohres: Herabsetzung des Hörvermögens für die Uhr auf $25-30 \mathrm{~cm}$, für Flüstersprache auf $5-6 \mathrm{~m}$, stark verkürzte Kopfknochenleitung, Verkürzung der Hördauer für hohe Töne, in geringem Grade für tiefe Töne.

Nach längerem Landaufenthalte, Einhaltung roborierender Diät und internem Gebrauche von Arsen kam das Kind bei bestem Wohlbefinden und ausgezeichnetem Aussehen wieder zur Untersuchung, wobei sich vollständig normale Verhältnisse ergaben. Wiederholte Nachprüfungen zeigten das gleiche Untersuchungsresultat.

XXIV. Therese K. 10 Jahre. Vor 2 Jahren Ikterus. Mit 6 Jahren Scarlatina. Häufig Kopfschmerzen, besonders in der linken Stirn- und Schläfegegend. Häufige Anfälle von Schwindel mit dem Gefühle des Drehens der Umgebung. Wiederholtes, oft sehr heftiges Ohrenstechen. Schlechtes Gedächtnis, schlechtes Auffassungsvermögen.

Rechtes Ohr normal. Linkes Ohr: Otoskopischer Befund normal. Hörweite für die Uhr $30 \mathrm{~cm}$, für Flüstersprache $6-8 \mathrm{~m}$. Weber nach rechts, Rinne beiderseits positiv. Schwabach rechts nomal, links leicht verkürzt. $c^{4}$ und $\mathrm{C}$ links verkürzt. Vestibularapparat: kein spontaner Nystagmus. Bei Drehung nach rechts 23 Sek., bei Drehung nach links 27 Sek. andauernder Nachnystagmus mit starkem Schwindel.

Gelegentlich eines Besuches der Ohrenabteilung zeigte das Kind nach elektriseher Behandlung die Erscheinungen eines starken Schwindelanfalles. Es war auffallend blaß, zeigte einen müden, ängstlichen Gesichtsausdruck, auffallende Unruhe, Angstgefühl und deutliche Unsicherheit beim Gehen. Es fand sich bei seitlicher Blickrichtung (sowohl nach rechts wie nach links) deutlicher kleinschlägiger horizontaler Nystagmus. Bei Drehung nach rechts 35 Sek., bei Drehung nach links 37 Sek. anhaltender Nachnystagmus mit starkem Schwindel.

Diagnose: Linksseitige geringgradige Erkrankung des inneren Ohres mit Reizerscheinungen von seiten des Vestibularapparates während eines Schwindelanfalles.

XXV. Ferdinand I. 12jähriger Kaufmannssohn. Allgemeine Müdigkeit, Zerstreutheit, geringes Auffassungsvermögen, Anfälle von Kopfschmerzen mit Schwindelgefühl und Ohrensausen, Appetitlosigkeit, Verstopfung, kolikartige Schmerzen im Unterleibe, Räuspern und Husten.

Trommelfell beiderseits normal. Uhr beiderseits $20-50 \mathrm{~cm}$, Flüstersprache $4-8 \mathrm{~m}$ (bei wiederholten Untersuchungen starke Differenzen). Schwabach immer stark verkürzt, ebenso $\mathbf{c}^{4}$ (Luftleitung). Exmüdungsphänomen beiderseits in wechselndem Grade, jedoch immer sehr deutlich ausgesprochen.

Diagnose: Beiderseitige geringgradige Erkrankung des inneren Ohres mit starken Schwankungen im Funktionsbefunde.

XXVI. Josef W. 11 Jahre. Nach linksseitiger alkuter eiteriger Mittelohrentzündung von vierwöchentlicher Dauer lästiges Summen in dem krankgewesenen $\mathrm{Ohr}$, das jedesmal nach längerer geistiger Beschäftigung oder körperlicher Anstrengung gleichzeitig mit Kopfschmerzen auftritt und dann stundenlang anhält.

Diagnose: Normales Gehör, normaler Funktionsbefund.

XXVII. Adolf L. 13 jähriger Gärtnergehilfenssohn. Kopfschmerzea, manchmal Schwindelgefühl, Gehör schwankend, häufig Surren in 
den Ohren, das an einzelnen Tagen bei schlechtem Allgemeinbefinden in heftiger Weise auftritt und stundenlang anhält.

Allgemeiner Status: Kornealreflexe und Rachenreflex fohlen, Sehnen- und Bauchdeckenreflexe gesteigert. Leichte Vergrößerung der Schilddruise. Hyperplasie des lymphatischen Rachenringes. Dermoid im rechten äußeren Augenwinkel, labiles Herz (Aschnerscher Bulbusdruckreflex, Erbensches Pulsphänomen, respiratorische Pulsirregularität). Links offener äuBerer Leistenring.

Eine Schwester augenkrank (Astigmatismus myopicus, Strabismus divergens alternans).

Ohrbefund: Trommelfell beiderseits normal. Uhr: rechts $30 \mathrm{~cm}$, links $25 \mathrm{~cm}$, Flüstersprache beiderseits $10 \mathrm{~m}$. Weber im Kopfe, Rinne positiv. Schwabach beiderseits verkürzt. $\mathrm{e}^{4}$ beiderseits 10 Sek. verkürzt. Deutliches Ermüdungsphänomen.

Diagnose: Beiderseitige geringgradige Erkrankung des inneren Ohres.

XXVIrI. Auguste Schm. 13 Jahre. Mutter seit 20 Jahren schwerhörig. Patientin soll bis vor 2 Jahren gut gehört haben. Seit jener Zeit langsame Gehörsabnahme ohne der Patientin bekannte Veranlassung. Keine Kinderkrankheiten. Zeitweise Kopfschmerzen, mitunter Ohrensausen. Wassermann positiv.

Trommelfell beiderseits normal. Uhr rechts $5 \mathrm{~cm}$, links o, Flïstersprache: rechts $1 \mathrm{~m}$, links o, laute Sprache rechts $+8 \mathrm{~m}$, links nur in einer Entfernung, innerhalb welcher das andere Ohr nicht ausgeschlossen werden kann. Akumeter rechts $1 \mathrm{~m}$, links o. Weber nach rechts, Rinne rechts positiv. Stenger links negativ. Vestibularapparat: Bei Drehung nach rechts 10 Sek. lang andauernde schwache Nystagmusschläge, bei Drehung nach links 20 Sek. andauernder starker Nachnystagmus. Kalorische Reaktion rechts positiv, links negativ. Kein Fistelsymptom. Kein spontaner Nystagmus.

Diagnose: Rechts mittelgradige Erkrankung des inneren Ohres, links Taubheit und Ausschaltung des Vestibularapparates.

XXIX. Rosa K. 131/2jährige Schneidermeisterstochter. Ein. Bruder des Vaters taubstumm. Patientin soll mit $6 \mathrm{Jahren}$ an Scharlach gelitten, darnach aber noch lange Zeit gut gehört haben. Erst $2-3$ Jahre später begann das Gehör abzunehmen. In der letzten Zeit öfters Sausen oder Klingen in den Ohren.

Trommelfellbefund beiderseits normal. Uhr: beiderseits ad concham. Flüstersprache: rechts $1 \frac{1 / 2}{\mathrm{~m}}$, links $1 \mathrm{~m}$. Laute Sprache : rechts $6 \mathrm{~m}$, links $6 \mathrm{~m}$. Weber im Kopt. Rinne beiderseits positiv. Schwabach beiderseits 20 Sek. verkürzt. $c^{4}$ rechts 15 , links 10 Sek. verkürzt, $O$ rechts 19 Sek., links 12 Sek. verkürzt. Beiderseits Ermüdungsphänomen. Vestibularapparat: Bei Drehung nach rechts 12 Sek. andauernder, bei Drehung nach links 16 Sek. andauernder Nachnystagmus. Kalorische Erregbarkeit beiderseits normal. Kein Aspirations- und Kompressionsnystagmus. Wassermann positiv.

Diagnose: Beiderseitige hochgradige Erkrankung des inneren Ohres.

XXX. Rudolf V. 7 Jahre. Bis zum Alter von 5 Jahren gesund; nach schwerer Influenza vor $13 / 4$ Jahren fortdauernder Appetitmangel, häufige Darmstörungen, schlechtes Aussehen. Nach längerem Landaufenthalt rorübergehende geringgradige Besserung.

Normales Gehör. Normalex Funktionsbefund.

XXXI. Beobachtung von Dr. Leidler; siehe Text S. 227 u. 228.

XXXII. Marie Kn. 10 Jahre. Zeitweise Kopfschmerzen. Manchmal bei heftigen Kopfschmerzen Klingen im Jinken Ohre. Mageres, blasses Mädchen. Keratitis parenchymatosa. Hutchinsonsche Zähne. Wassermann positiv. 
Ohrbefund: Normale Trommelfelle. Hörvermögen rechts: Uhr $40 \mathrm{~cm}$, Flüstersprache $8-10 \mathrm{~m}$, links: $U h r 10 \mathrm{~cm}$, Flüstersprache 4 bis $7 \mathrm{~m}$. (Bei wiederholten Untersuchungen ziemlich beträchtliche Differenzen). Weber im Kopf. Rinne beiderseits positiv. Schwabach rechts in geringem Grade (5 Sek.), links 10-15Sek. verkürzt. $e^{4}$ rechts normal, links 10 Sek. verkürzt. Antiluetische Behandlung ( $\mathrm{Hg}$ und Jod) ohne Erfolg.

Diagnose: Rechts geringgradige, links mittelgradige Erkrankung des inneren Ohres.

XXXIII. Gustav K. 11 jähriger Buchhalterssohn. Eine Schwester schwerhörig. Übrige Familienanamnese belanglos.

Allgemeiner Status: Asymmetrie des Gesichtes. Chvostek +, Thymusdämpfung (?), Rachenreflex und Kormealreflexe herabgesetzt. Sehnen- und Bauchdeckenreflexe beiderseits gesteigert. Cor sehr labil, systolisches Geräusch, schwach, über der Pulmonalis. Dermographismus. Orines pubis auffallend stark entwickelt.

Ohrbefund: Trommelfell beiderseits normal. Uhr: rechts $40-$ $50 \mathrm{~cm}$, links $23-30 \mathrm{~cm}$. Flüstersprache rechts $6-8 \mathrm{~m}$, links $4-6 \mathrm{~m}$. Weber im Kopf, Rinne beiderseits positiv. Kopfknochenleitung beiderseits verkürt. $c^{4}$ (Luftleitung) verkürzt. Befund bei Untersuchungen an verschiedenen Tagen stark differierend.

Diagnose: Rechts geringgradige, links mittelgradige Erkrankung des inneren Ohres.

XXXIV. Emmerich R. 12jähriger Kaufmannssohn. Großvater väterlicherseits, 9 Jahre gelähmt, erlag einem Schlaganfalle, Großmutter mütterlicherseits im Alter von 42 Jahren an Magenkrebs, Großvater mütterlicherseits an einem Herzleiden gestorben. Mutter nierenkrank, hört seit Jahren schlecht (labyrinthäre Schwerhörigkeit). Eine Schwester und ein Bruder des Vaters an Tuberkulose gestorben. Ein Bruder des Patienten ist an Gehirnhautentzündung gestorben, eine Schwester des Patienten leidet an Tuberkulose.

Patient schläft schlecht, leidet oft an Bauchschmerzen und Diarrhoe. Gehör in der letzten Zeit weniger scharf. Mitunter Summen in den Ohren, das oft stundenlang anhält.

Allgemeiner Status: AsthenischerHabitus. Apicitis bilateralis. Spitzbogengaumen. Hyperplasie des lymphatischen Rachenringes. Kornealreflexe und Rachenreflex fehlen, Periost- und Sehnenreflexe gesteigert. Dermographismus. Labiles Herz. Drïsenschwellung am Halse.

Ohrbefund: Trommelfelle normal. Uhr: beiderseits $30 \mathrm{~cm}$. Flüstersprache rechts $8-10 \mathrm{~m}$, links $8 \mathrm{~m}$. Weber unbestimmt, Rinne beiderseits positiv. Schwabach verkürzt. $e^{4}$ und $\mathrm{C}$ verkürzt. Sehr deutliches Ermü̈dungsphänomen. Vestibularapparat normal.

Diagnose: Beiderseitige geringgradige Erkrankung des inneren ohres.

XXXV. Leopold $\mathrm{S}$. 13 jähriger Dienstmannssohn. Großvater mütterlicherseits: Rheumatismus, Herzleiden. Großmuiter mütterlicherseits leberleidend. Mutter leidet an Gallensteinen und an leichtgradiger labyrinthärer Schwerhörigkeit. Vater ist Alkoholiker. Ein Bruder der Mutter Epileptiker, ein Bruder des Vaters schwachsinnig. Eine Schwester des Patienten (17 Jahre alt) Struma parenchymatosa. Hört seit ca. 2 Jahren schlechter und leidet an zeitweise wiederkehrenden Ohrgeräuschen (Rauschen oder Sausen). Oft Kopfschmerzen.

Allgemeiner Status: Synophris. Angewachsene Ohrläppchen. Hoher Gaumen. Rachenreflex und Kornealreflexe fehlen, Sehnenreflexe und Bauchdeckenreflexe stark gesteigert. Patellarreflexe sehr lebhaft. Rigide Art. rad, et temp. Dermographismus rot, sehr intensiv. Respiratorische Irregularität. Erbensches Pulsphänomen. Scap. scaphoid. Crines pub. weiblich, gut entwickelt. Auffallead unproportionierter enormer Penis. 
Ohrbefund: Uhr: rechts $30 \mathrm{~cm}$, links $50 \mathrm{~cm}$. Flüstersprache rechts $8-10 \mathrm{~m}$, links $7-8 \mathrm{~m}$. Weber im Kopf. Rinne positiv, Schwabach leicht verkürzt. $c^{4}$ leicht verkürzt. Beiderseits Frmüdungsphänomen.

Diagnose: Beiderseitige leichtgradige Erkrankung des inneren Ohres.

XXXVI, Robert Schw. 10jähriger Goldarbeiterssohn. Kopfschmerzen, leichte Ermüdbarkeit, schlechtes Auffassungsvermögen, unruhiger Schlaf. Schnarcht nachts. Hörvermögen seit kurzem stark reduziert. Trommelfelle retrahiert. Hörvermögen beiderseits wesentlich herabgesetzt (Flüstersprache rechts $1 / 2 \mathrm{~m}$, links $1 \mathrm{~m}$ ). Nach Luftdusche bedeutende Gehörverbesserung. Adenoide Vegetationen. Hypertrophia tonsillarum. Beiderseits Ermüdungsphänomen.

Diagnose: Bejderseitiger acuter Mit tolobrkatarrh.

XXXVII. Heinrich G. 9 Jahre. Auffallend blaß, schwächlich. Sehr zerstreut. Allgemeine Unruhe (Zappeln, Zupfen). Sehr kihle, feuchte, bläuliche Hände. Schwitzt sehr stark. Oft Kopfschmerzen. Drüsenschwellung am Halse.

Normales Gehör. Funktionsbefund normal, bis auf sehr deutlich ausgesprochenes Ermüdungsphänomen.

XXXVIII. Konrad Sch. 12 Jahre. Klagt über zeitweise auftretende Schmerzen in den Ohren. Häufige Kopfschmerzen (Gefuhl eines Reifens um den Kopf).

Gehör normal. Funktionsbefund normal, bis auf eklatantes Ermüdungsphänomen.

XXXIX. Anna Br. 12 Jahre. Vater sehr nervös und reizbar. Mutter blutarm, unterernährt. Zwei Geschwister sehr zart und blaB. Patientin klein, außerordentlich zart, klagt über häufig wiederkehrende, anfallsweise und dann oft stundenlang andauernde Kopfschmerzen. Seit einem halben Jahre merkt die Umgebung des Kindes, daß dasselbe weniger gut hört. Schwindel und Ohrensausen haben nie bestanden. Wassermann positiv.

Ohrbefund: Trommelfell beiderseits normal. Uhr: rechts $30 \mathrm{~cm}$, links 0 , Flüstersprache rechts $8-10 \mathrm{~m}$, links 0 , la ute Sprache rechts $+10 \mathrm{~m}$, links in einer Entfernung, innerhalb welcher das andere Ohr nicht ausgeschlossen werden kann. Akumeter rechts $8 \mathrm{~m}$, links 0 .

Rinne beiderseits positiv, Weber nach rechts. Schwabach in geringem Grade verkürzt. $c^{4}$ rechts verkürzt, links 0 . Vestibularapparat : kein spontaner Nystagmus. Bei zehn Drehungen nach links 18 Sek. andanernder deutlicher Nachnystagmus, bei zehn Drehungen nach rechts $6-7$ Sek. andauernde kleine Nystagmusschläge. Kalorische $\mathbf{E r}-$ regbarkeit rechts normal, links 0 . Kein Schwindel, keine Gleichgewichtsstörung. Kein Aspirations-, und Kompressionsnystagmus.

Diagnose: Rechts: geringgradige Erkrankung des inneren Ohres; links: Taubheit und Ausschaltung des Vestibularapparates.

XXXX. Elisabeth Sch. \& Jahre. Leichte Frmüdbarkeit, anfallsweise auBerordentlich fahle Blässe des Gesichts. Nervöses Räuspern und Hüsteln. Allgemeine Unruhe (Zappeln, Nägelkauen, Zupfen usw.) Häufig starke Kopfschmerzen und Schwindelgefühl. Hört seit einigen Tagen nach starkem Schnupfen auffallend schlecht. Wassermann positiv (auch bei der Mutter des Kindes).

Ohrbefund: Trommelfell: links stark retrahiert, in der Trommelhöhle seröses Transsudat; rechts normal. Uhr: links $5 \mathrm{~cm}$, rechts 0 , Flüstersprache links $2 \mathrm{~m}$, rechts 0 , laute Splache rechts $8 \mathrm{~m}$, links 0 . (Nach Luftdusche links Uhr $35 \mathrm{~cm}$, Flüstersprache $8 \mathrm{~m}$, laute Sprache $+10 \mathrm{~m}$.) Weber unbestimmt, Rinne links positiv, Schwabach etwas verkürzt. Vestibularapparat: Kein spontaner Nystagmus. Kein Schwindel, keine Gleichgewichtsstörung.

Untersuchung auf dem Drehstuhle: Nach 10 Drehungen nach 
rechts 12 Sek. andauernder deutlicher Nachnystagmus, nach 10 Drehungen nach links einige Sekunden anhaltende schwache nystaktische Zuckungen. Kalorische Erregbarkeit links normal, rechts bedeutend. vermindert (erst nach $3 / 41$ kalten Wassers in geringem Grade auslösbar. Kein Nystagmus bei Aspiration und Kompression.

Diagnose: Links: Akuter sekretorischer Mittelohrkatarrh und leichtgradige Erkrankung des inneren Ohres. Rechts: Taubheit und stark verminderte Reflexerregbarkeit des Vestibularapparates.

\section{Literatur.}

Alexander, G. Die Ohrenkrankheiten im Kindesalter. Leipzig, Verlag von F. C. W. Vogel, 1912.

Baner, J., und Stein, C. Die Bedeutung der Konstitution in der Pathogenese der Otosklerose. Zeitschr. f. angewandte Anatomie und Konstitutionslehre. 1914. Bd. I, Heft 6 .

Bryant, Sohier. Die Ätiologie der Otosklerose. (Osteodystrophia petrosa Bryant.) Monatsschr. f. Ohrenh. 1913, Nr. 3 u. 4.

Burnett, zit. nach Urbantschitsch. Lehrbuch d. Ohrenheilkunde. Delmarcel und Delstanche. Kongr. d. belg. Gesellsch. f. Otol. u. Rhin. Brüssel, 13. bis 14. Juli 1912. Zit. nach Zentralbl, f. Ohrenh. 1912.

Hamburger. Ủber Arterienrigidität im Kindesalter. Münchener med. Wochenschrift 1911, Nr. 5 .

- Übex den vasoneurotischen Symptomenkomplex bei Kindern. Münchener med. Wochenschrift $1911, \mathrm{Nr} .42$.

Hammers chlag. Zur Diagnose der funktionellen Erkrankungen des schallperzip. Apparates. Allg. Wiener med. Zeitg. Bd. 49, S. 45 und 46 .

Itard, zit. nach Urbantschitsch. Lehrb. d. Ohrenh.

Krehl, zit. nach Hamburger.

Manasse, zit. nach Alexander.

Mayer, O. Die Pathogenese und Ätiologie d. Otosklerose. Monatsschr. f. Ohrenh. 1911.

Politzer. Lehrb. d. Ohrenheilkunde. 5. Aufl, 1904.

Romberg, zit. nach Hamburger.

Schlayer, zit. nach Hamburger.

Stein, C. Otalgia angiosclerotica. Ein Beitrag z. Pathogenese der Schmerzempfindungen im Bereiche des Gehörorganes. Wiener klin. Wochenschrift 1912, Nr. 26.

- Über die ZirkulationsverhäItnisse im Schädelinneren bei venöser Stauung und ihre Beziehungen zu den Ohrgeräuschen. Arch. f. Ohrenheilk. 1911, Bd. 86.

- Die klinische Pathologie und Therapie der subj. Ohrgeräusche. Monatsschrift f. Ohrenheilk. 1914. 48. Jahrg., Nr. 5.

Urbantschitsch, V. Lehrbuch der Ohrenheilkunde. 5. Aufl. 1910. Wendt, zit. nach Urbantschitsch. Lehrb. d. Ohrenheilkunde.

Wittmaack. Die toxische Neuritis acustica. Zeitschr. f. Ohrenh. Bd. 46 . 